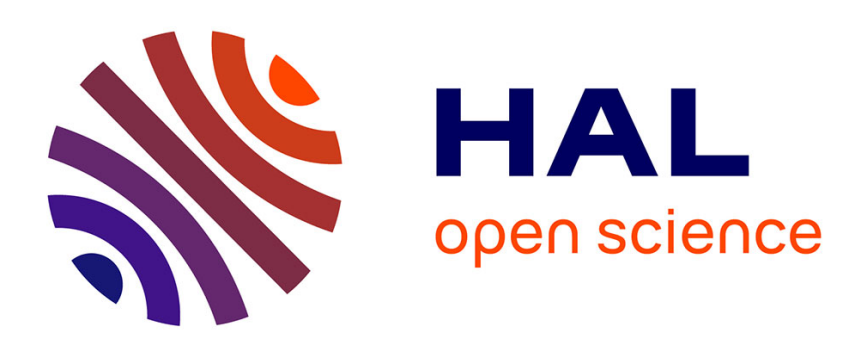

\title{
On the influence of the geometry on skin effect in electromagnetism
}

\author{
Gabriel Caloz, Monique Dauge, Erwan Faou, Victor Péron
}

\section{To cite this version:}

Gabriel Caloz, Monique Dauge, Erwan Faou, Victor Péron. On the influence of the geometry on skin effect in electromagnetism. Computer Methods in Applied Mechanics and Engineering, 2011, 200 (9-12), pp.1053-1068. 10.1016/j.cma.2010.11.011 . hal-00503170v2

HAL Id: hal-00503170 https://hal.science/hal-00503170v2

Submitted on 1 Nov 2010

HAL is a multi-disciplinary open access archive for the deposit and dissemination of scientific research documents, whether they are published or not. The documents may come from teaching and research institutions in France or abroad, or from public or private research centers.
L'archive ouverte pluridisciplinaire HAL, est destinée au dépôt et à la diffusion de documents scientifiques de niveau recherche, publiés ou non, émanant des établissements d'enseignement et de recherche français ou étrangers, des laboratoires publics ou privés. 


\title{
ON THE INFLUENCE OF THE GEOMETRY ON SKIN EFFECT IN ELECTROMAGNETISM
}

\author{
GABRIEL CALOZ, MONIQUE DAUGE, ERWAN FAOU, VICTOR PÉRON
}

\begin{abstract}
We consider the equations of electromagnetism set on a domain made of a dielectric and a conductor subdomain in a regime where the conductivity is large. Assuming smoothness for the dielectric-conductor interface, relying on recent works we prove that the solution of the Maxwell equations admits a multiscale asymptotic expansion with profile terms rapidly decaying inside the conductor. This skin effect is measured by introducing a skin depth function that turns out to depend on the mean curvature of the boundary of the conductor. We then confirm these asymptotic results by numerical experiments in various axisymmetric configurations. We also investigate numerically the case of a nonsmooth interface, namely a cylindrical conductor.
\end{abstract}

\section{CONTENTS}

1. Introduction 1

2. Framework 3

3. Multiscale expansion 5

4. Axisymmetric domains 7

5. Finite element discretizations and computations 13

6. Numerical simulations of skin effect 17

7. Postprocessing 21

8. Conclusion 25

Appendix A. Elements of proof for the multiscale expansion 25

Appendix B. The multiscale expansion of the orthoradial component 33

References $\quad 36$

\section{INTRODUCTION}

Our interest lies in the influence of the geometry of a conducting body on the skin effect in electromagnetism. This effect describes the rapid decay of electromagnetic fields with depth inside a metallic conductor. The skin effect reflects the flow of current near the surface of a conductor. After the early work [18], the mathematical analysis of the skin effect has been addressed more recently in several papers, $[20,11,12,8]$.

The present work is motivated by recent studies $[8,4,3,17]$ in which authors analyze the behavior of the electromagnetic fields solution of the Maxwell equations through an asymptotic expansion for large conductivity. In particular, uniform estimates for the 
electromagnetic field at high conductivity are proved in [3], whereas in the note [4] a suitable skin depth function is introduced on the interface between a conductor and an insulator to generalize the classical scalar quantity. An asymptotic expansion at high conductivity for this function shows the influence of the geometry of the interface : the skin depth is larger for high conductivity when the mean curvature of the conducting body surface is larger - and here the sign of the curvature has a major influence, which means that the skin depth is larger in convex than in concave conductors.

In this paper, our aim is twofold

(1) Present elements of derivation for asymptotic expansions near the conductorinsulator interface,

(2) Illustrate by numerical computations the theoretical behavior deduced from asymptotic analysis in [4].

For our computations, we consider a special class of axisymmetric problems, which allows their reduction to two-dimensional scalar problems. This enables to measure the skin effect with reduced computational effort. We perform finite element computations for relevant benchmarks (cylinders and spheroids). The interface problem for the magnetic field is solved with the library Mélina [13]. A postprocessing shows the accuracy of the asymptotic expansion, and exhibits the influence of the mean curvature of the conductor on the skin effect. Our computations also clearly display the effect of the edges of the cylindrical conductor: In this case, the decay is not exponential near edges while becoming exponential further away from edges.

The presentation of the paper proceeds as follows. In section 2, we introduce the framework: we present the Maxwell equations and the result of [3] about existence of solution at high conductivity and the formulation for the magnetic field. In section 3 , we present the asymptotic expansion of the magnetic field in the smooth case, and the asymptotic behavior of the skin depth for high conductivity. In section 4, we restrict our considerations to axisymmetric domains and orthoradial axisymmetric data, and we present the configurations $\mathrm{A}$ (cylindrical), $\mathrm{B}$, and $\mathrm{C}$ (spheroidal), chosen for computations. In section 5, we introduce a finite element discretization for the solution of the problem in the meridian domain, and we check the convergence of the discretized problem. In section 6, we present numerical simulations in all configurations to highlight the skin effect, and to exhibit the influence of the mean curvature of the conductor on the skin effect. In section 7, we perform post-treatments of our numerical computations in configurations A (cylindrical) and B (spheroidal) in order to investigate the nature of the decay of the field inside the conductor: In smooth configurations (B) the rate of the exponential decay is very close to the expected theoretical one, while in corner configurations (A) the exponential decay shows up in a region which is not very close to the corner. Theoretical aspects in this latter case are going to be studied in [5]. In Appendix A, we provide elements of proof for the multiscale expansion given in section 3 . We expand the Maxwell operators in power series, and we obtain the equations satisfied by 
the magnetic and electric profiles. In Appendix B, we derive the profiles of the orthoradial component of the magnetic field in relation with the $3 \mathrm{D}$ asymptotic expansion in axisymmetric configurations.

\section{FRAMEWORK}

Let $\Omega$ be a piecewise smooth Lipschitz domain. We denote by $\Gamma$ its boundary and by $\mathbf{n}$ the outer unit normal field on $\Gamma$. We denote by $\mathbf{L}^{2}(\Omega)$ the space of three-component fields square integrable on $\Omega$.

We consider the Maxwell equations given by Faraday's and Ampère's laws in $\Omega$ :

$$
\operatorname{curl} \mathbf{E}-i \omega \mu_{0} \mathbf{H}=0 \text { and } \operatorname{curl} \mathbf{H}+\left(i \omega \varepsilon_{0}-\underline{\sigma}\right) \mathbf{E}=\mathbf{j} \quad \text { in } \Omega \text {. }
$$

Here, $(\mathbf{E}, \mathbf{H})$ represents the electromagnetic field, $\mu_{0}$ is the magnetic permeability, $\varepsilon_{0}$ the electric permittivity, $\omega$ the angular frequency, $\mathbf{j}$ represents a current density and is supposed to belong to

$$
\mathbf{H}_{0}(\operatorname{div}, \Omega)=\left\{\mathbf{u} \in \mathbf{L}^{2}(\Omega) \mid \operatorname{div} \mathbf{u} \in \mathrm{L}^{2}(\Omega), \mathbf{u} \cdot \mathbf{n}=0 \text { on } \Gamma\right\},
$$

and $\underline{\sigma}$ is the electric conductivity. We assume that the domain $\Omega$ is made of two (connected) subdomains $\Omega_{+}$and $\Omega_{-}$in which the coefficient $\underline{\sigma}$ take two different values $\left(\sigma_{+}=0, \sigma_{-} \equiv \sigma\right)$. We denote by $\Sigma$ the interface between the subdomains $\Omega_{+}$and $\Omega_{-}$. In the most part of our analysis ${ }^{1}$, we will assume that the external boundary $\Gamma$ is contained in the dielectric region $\bar{\Omega}_{+}$, see Figure 1 .

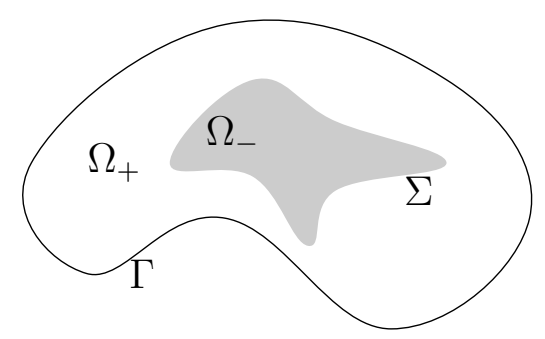

FIGURE 1. The domain $\Omega$ and its subdomains $\Omega_{+}$(dielectric) and $\Omega_{-}$(conductor).

To complement the Maxwell harmonic equations (2.1), we consider the perfectly insulating electric boundary conditions on the boundary $\Gamma$

$$
\mathbf{E} \cdot \mathbf{n}=0 \text { and } \mathbf{H} \times \mathbf{n}=0 \quad \text { on } \Gamma .
$$

Note that we could also consider the perfectly conducting electric boundary conditions $\mathbf{E} \times \mathbf{n}=0$ and $\mathbf{H} \cdot \mathbf{n}=0$ on $\Gamma$.

\footnotetext{
${ }^{1}$ Except in one configuration in which the roles of the conductor and dielectric bodies are swapped.
} 
2.1. Existence of solutions. Subsequently, we assume that the following condition on the limit problem in the dielectric part $\Omega_{+}$is valid:

Assumption 2.1. The angular frequency $\omega$ is not an eigenfrequency of the problem

$$
\begin{cases}\operatorname{curl} \mathbf{E}-i \omega \mu_{0} \mathbf{H}=0 \quad \text { and } \quad \operatorname{curl} \mathbf{H}+i \omega \varepsilon_{0} \mathbf{E}=0 & \text { in } \Omega_{+} \\ \mathbf{E} \times \mathbf{n}=0 \quad \text { and } \mathbf{H} \cdot \mathbf{n}=0 & \text { on } \Sigma \\ (2.2) & \text { on } \Gamma .\end{cases}
$$

Hereafter, we denote by $\|\cdot\|_{0, \mathcal{O}}$ the norm in $\mathbf{L}^{2}(\mathcal{O})$. We quote from [3, Th.2.3]:

Theorem 2.2. If the interface $\Sigma$ is Lipschitz, under Assumption 2.1, there are constants $\sigma_{0}$ and $C>0$, such that for all $\sigma \geqslant \sigma_{0}$, the Maxwell problem (2.1) with boundary condition (2.2) and data $\mathbf{j} \in \mathbf{H}_{0}(\operatorname{div}, \Omega)$ has a unique solution $(\mathbf{E}, \mathbf{H})$ in $\mathbf{L}^{2}(\Omega)^{2}$, which satisfies:

$$
\|\mathbf{E}\|_{0, \Omega}+\|\mathbf{H}\|_{0, \Omega}+\sqrt{\sigma}\|\mathbf{E}\|_{0, \Omega_{-}} \leqslant C\|\mathbf{j}\|_{\mathbf{H}(\operatorname{div}, \Omega)} .
$$

For convenience, we introduce in the Maxwell equations (2.1) the small parameter

$$
\delta=\sqrt{\omega \varepsilon_{0} / \sigma} .
$$

Hence, $\delta$ tends to 0 when $\sigma \rightarrow \infty$. For $\sigma \geqslant \sigma_{0}$, we denote by $\left(\mathbf{E}_{(\delta)}, \mathbf{H}_{(\delta)}\right)$ the solution of the system (2.1) $-(2.2)$.

2.2. Magnetic formulation. By a standard procedure we deduce from the Maxwell system (2.1)-(2.2) the following variational formulation for the magnetic field $\mathbf{H}_{(\delta)}$. The variational space is $\mathbf{H}_{0}(\operatorname{curl}, \Omega)$ :

$$
\mathbf{H}_{0}(\operatorname{curl}, \Omega)=\left\{\mathbf{u} \in \mathbf{L}^{2}(\Omega) \mid \operatorname{curl} \mathbf{u} \in \mathbf{L}^{2}(\Omega), \mathbf{u} \times \mathbf{n}=0 \text { on } \Gamma\right\},
$$

and the variational problem writes

Find $\mathbf{H}_{(\delta)} \in \mathbf{H}_{0}(\operatorname{curl}, \Omega)$ such that for all $\mathbf{K} \in \mathbf{H}_{0}(\operatorname{curl}, \Omega)$

$$
\int_{\Omega}\left(\frac{1}{\varepsilon(\delta)} \operatorname{curl} \mathbf{H}_{(\delta)} \cdot \operatorname{curl} \mathbf{K}-\kappa^{2} \mathbf{H}_{(\delta)} \cdot \mathbf{K}\right) \mathrm{d} \mathbf{x}=\int_{\Omega}\left(\operatorname{curl} \frac{\mathbf{j}}{\varepsilon(\delta)}\right) \cdot \mathbf{K} \mathrm{d} \mathbf{x}
$$

where we have set

$$
\varepsilon(\delta)=\mathbf{1}_{\Omega_{+}}+\left(1+\frac{i}{\delta^{2}}\right) \mathbf{1}_{\Omega_{-}} \quad \text { and } \quad \kappa:=\omega \sqrt{\varepsilon_{0} \mu_{0}} .
$$

Assumption 2.3. We assume that the surfaces $\Sigma$ (interface) and $\Gamma$ (external boundary) are smooth.

Under Assumption 2.3, the magnetic field $\mathbf{H}_{(\delta)}$ is globally in $\mathbf{H}^{1}(\Omega)$. 


\section{Multiscale expansion}

Several works are devoted to asymptotic expansions at high conductivity of the electromagnetic field in a domain made of two subdomains when the interface is smooth: see $[20,11,12]$ for plane interface and eddy current approximation, and [8, 3, 4, 17] for a three-dimensional model of skin effect in electromagnetism.

In this section we recall the results presented in [4] on the behavior of the skin depth function. This relies on asymptotic expansions for the electromagnetic field analyzed in the $\mathrm{PhD}$ thesis [17]. For the sake of completeness we give in Appendix A elements of proofs.

Here, we assume that $\mathbf{j}$ is smooth and that Assumptions 2.1 and 2.3 hold. Let $\mathcal{U}_{-}$be a tubular neighborhood of the surface $\Sigma$ in the conductor part $\Omega_{-}$, see Figure 2 . We denote by $\left(y_{\alpha}, y_{3}\right)$ a local normal coordinate system to the surface $\Sigma$ in $\mathcal{U}_{-}$: Here, $y_{\alpha}, \alpha=1,2$, are tangential coordinates on $\Sigma$ and $y_{3}$ is the normal coordinate to $\Sigma$, cf. [14, 6].

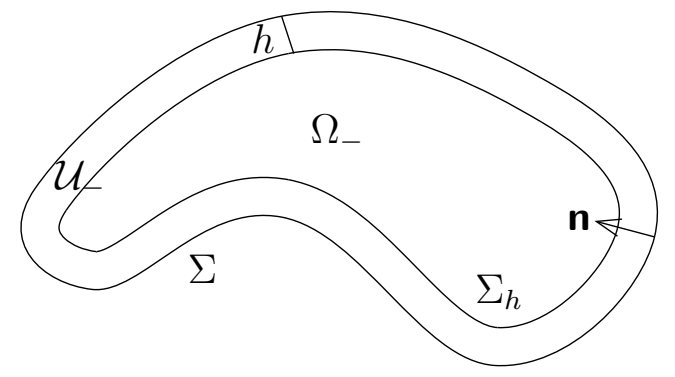

FIGURE 2. A tubular neighbourhood of the surface $\Sigma$

In view of our numerical computations, we concentrate on the magnetic field $\mathbf{H}_{(\delta)}$ solution of (2.7). It is denoted by $\mathbf{H}_{(\delta)}^{+}$in the dielectric part $\Omega_{+}$, and by $\mathbf{H}_{(\delta)}^{-}$in the conducting part $\Omega_{-}$. Both parts exhibit series expansions in powers of $\delta$ :

$$
\begin{gathered}
\mathbf{H}_{(\delta)}^{+}(\mathbf{x})=\mathbf{H}_{0}^{+}(\mathbf{x})+\delta \mathbf{H}_{1}^{+}(\mathbf{x})+\mathcal{O}\left(\delta^{2}\right), \quad \mathbf{x} \in \Omega_{+} \\
\mathbf{H}_{(\delta)}^{-}(\mathbf{x})=\mathbf{H}_{0}^{-}(\mathbf{x} ; \delta)+\delta \mathbf{H}_{1}^{-}(\mathbf{x} ; \delta)+\mathcal{O}\left(\delta^{2}\right), \quad \mathbf{x} \in \Omega_{-} \\
\text {with } \quad \mathbf{H}_{j}^{-}(\mathbf{x} ; \delta)=\chi\left(y_{3}\right) \underline{\mathfrak{H}}_{j}\left(y_{\beta}, \frac{y_{3}}{\delta}\right) .
\end{gathered}
$$

In (3.1)-(3.2), the symbol $\mathcal{O}\left(\delta^{2}\right)$ means that the remainder is uniformly bounded by $\delta^{2}$, and in (3.3), the function $\mathbf{y} \mapsto \chi\left(y_{3}\right)$ is a smooth cut-off with support in $\overline{\mathcal{U}}_{-}$and equal to 1 in a smaller tubular neighborhood of $\Sigma$. The vector fields $\underline{\mathfrak{Y}}_{j}:\left(y_{\alpha}, Y_{3}\right) \mapsto \underline{\mathfrak{Y}}_{j}\left(y_{\alpha}, Y_{3}\right)$ are profiles defined on $\Sigma \times \mathbb{R}^{+}$: They are exponentially decreasing with respect to $Y_{3}$ and are smooth in all variables.

3.1. First terms of asymptotics in the conductor region. Hereafter, we present the construction of the first profiles $\underline{\mathfrak{Y}}_{j}=\left(\mathfrak{H}_{j}^{\alpha}, \mathfrak{h}_{j}\right)$ and of the first terms $\mathbf{H}_{j}^{+}$. The normal 
component $\mathfrak{h}_{0}$ of the first profile in the conductor is zero:

$$
\mathfrak{h}_{0}=0 \text {. }
$$

Then, the first term of the magnetic field in the dielectric region solves Maxwell equations with perfectly conducting conditions on $\Sigma$ :

$$
\begin{cases}\operatorname{curl} \operatorname{curl} \mathbf{H}_{0}^{+}-\kappa^{2} \mathbf{H}_{0}^{+}=\operatorname{curl} \mathbf{j} & \text { in } \Omega_{+} \\ \mathbf{H}_{0}^{+} \cdot \mathbf{n}=0 \text { and } \operatorname{curl} \mathbf{H}_{0}^{+} \times \mathbf{n}=0 & \text { on } \Sigma \\ \mathbf{H}_{0}^{+} \times \mathbf{n}=0 \text { and } \operatorname{div} \mathbf{H}_{0}^{+}=0 & \text { on } \Gamma .\end{cases}
$$

Thus the trace $\mathbf{h}_{0}$ of $\mathbf{H}_{0}^{+}$on the interface $\Sigma$ is tangential.

The first profile in the conductor region is exponential with the complex rate $\lambda$ such that $\lambda^{2}=-i \kappa^{2}$ :

$$
\underline{\mathfrak{Y}}_{0}\left(y_{\beta}, Y_{3}\right)=\mathbf{h}_{0}\left(y_{\beta}\right) \mathrm{e}^{-\lambda Y_{3}} \quad \text { with } \quad \lambda=\kappa \mathrm{e}^{-i \pi / 4} \text {. }
$$

Note that, if $\mathbf{h}_{0}$ is not identically 0 , there exists $C_{0}>1$ independent of $\delta$ such that

$$
C_{0}^{-1} \sqrt{\delta} \leqslant\left\|\mathbf{H}_{0}^{-}(\cdot ; \delta)\right\|_{0, \Omega_{-}} \leqslant C_{0} \sqrt{\delta} .
$$

The next term which is determined in the asymptotics is the normal component $\mathfrak{h}_{1}$ of the profile $\underline{\mathfrak{H}}_{1}$ :

$$
\mathfrak{h}_{1}\left(y_{\beta}, Y_{3}\right)=\lambda^{-1} D_{\alpha} \mathrm{h}_{0}^{\alpha}\left(y_{\beta}\right) \mathrm{e}^{-\lambda Y_{3}}
$$

Here $D_{\alpha}$ is the covariant derivative on $\Sigma$ and we use the summation convention of repeated indices. The next term in the dielectric region solves:

$$
\begin{cases}\text { curl curl } \mathbf{H}_{1}^{+}-\kappa^{2} \mathbf{H}_{1}^{+}=0 & \text { in } \Omega_{+} \\ \mathbf{H}_{1}^{+} \cdot \mathbf{n}=\mathfrak{h}_{1} \quad \text { and } \quad \operatorname{curl} \mathbf{H}_{1}^{+} \times \mathbf{n}=i \lambda \mathbf{h}_{0} & \text { on } \Sigma \\ \mathbf{H}_{1}^{+} \times \mathbf{n}=0 \quad \text { and } \quad \operatorname{div} \mathbf{H}_{1}^{+}=0 & \text { on } \Gamma .\end{cases}
$$

Like above, $\mathbf{h}_{1}$ is the trace of $\mathbf{H}_{1}^{+}$on the interface $\Sigma$, and $\mathrm{h}_{1}^{\alpha}$ denote its tangential components. The tangential components $\mathfrak{H}_{1}^{\alpha}$ of the profile $\underline{\mathfrak{Y}}_{1}$ are given by

$$
\mathfrak{H}_{1}^{\alpha}\left(y_{\beta}, Y_{3}\right)=\left[\mathrm{h}_{1}^{\alpha}+Y_{3}\left(H \mathrm{~h}_{0}^{\alpha}+b_{\sigma}^{\alpha} \mathrm{h}_{0}^{\sigma}\right)\right]\left(y_{\beta}\right) \mathrm{e}^{-\lambda Y_{3}}, \quad \alpha=1,2 .
$$

Here $b_{\alpha}^{\gamma}=a^{\gamma \beta} b_{\beta \alpha}$, where $a^{\gamma \beta}$ is the inverse of the metric tensor $a_{\alpha \beta}$ in $\Sigma$, and $b_{\alpha \beta}$ is the curvature tensor in $\Sigma$ and

$$
H=\frac{1}{2} b_{\alpha}^{\alpha}
$$

is the mean curvature of the surface $\Sigma$. In particular, the sign of $H$ depends on the orientation of the surface $\Sigma$. As a convention, the unit normal vector $\mathbf{n}$ on the surface $\Sigma$ is inwardly oriented to $\Omega_{-}$, see Figure 2 . 
3.2. Asymptotic behavior of the skin depth. In a one-dimensional model, when the conductor $\Omega_{-}$is a half-space, the classical skin depth parameter is given by

$$
\ell(\sigma)=\sqrt{\frac{2}{\omega \mu_{0} \sigma}} .
$$

This length corresponds to the distance from the surface of the conductor where the field has decreased of a rate e. In our situation, following [4, def. 4.1], we extend this definition to curved interfaces. For a data $\mathbf{j}$, let us define

$$
\underline{\mathfrak{H}}_{(\delta)}\left(y_{\alpha}, y_{3}\right):=\mathbf{H}_{(\delta)}^{-}(\mathbf{x}), \quad y_{\alpha} \in \Sigma, \quad 0 \leqslant y_{3}<h_{0},
$$

for $h_{0}$ small enough. Hereafter for any $\mathbf{Z}=\left(z_{1}, z_{2}, z_{3}\right) \in \mathbb{C}^{3},|\mathbf{Z}|$ denotes the vectornorm $\left(\left|z_{1}\right|^{2}+\left|z_{2}\right|^{2}+\left|z_{3}\right|^{2}\right)^{1 / 2}$ in $\mathbb{C}^{3}$ and $\langle\cdot, \cdot\rangle$ the corresponding hermitian scalar product.

Definition 3.1. Let $\Sigma$ be a smooth surface, and $\mathbf{j}$ a data of problem (2.1). such that for all $y_{\alpha}$ in $\Sigma, \underline{\mathfrak{T}}_{(\delta)}\left(y_{\alpha}, 0\right) \neq 0$. The skin depth is the length $\mathcal{L}\left(\sigma, y_{\alpha}\right)$ defined on $\Sigma$ and taking the smallest positive value such that

$$
\left|\underline{\mathfrak{Y}}_{(\delta)}\left(y_{\alpha}, \mathcal{L}\left(\sigma, y_{\alpha}\right)\right)\right|=\left|\underline{\mathfrak{H}}_{(\delta)}\left(y_{\alpha}, 0\right)\right| \mathrm{e}^{-1} .
$$

Thus the length $\mathcal{L}\left(\sigma, y_{\alpha}\right)$ is the distance from the interface where the field has decreased of a fixed rate. It depends on the conductivity $\sigma$ and of each point $y_{\alpha}$ in the interface $\Sigma$. A priori it also depends on the data $\mathbf{j}$.

As a consequence of (3.5) and (3.9), there holds

$$
\left\{\begin{array}{l}
\left|\underline{\mathfrak{T}}_{(\delta)}\left(y_{\alpha}, y_{3}\right)\right|^{2}=\left|\mathbf{h}_{0}\left(y_{\alpha}\right)\right|^{2} \mathfrak{m}\left(y_{\alpha}, y_{3} ; \delta\right) \mathrm{e}^{-2 y_{3} \operatorname{Re}(\lambda) / \delta}, \quad \text { with } \\
\mathfrak{m}\left(y_{\alpha}, y_{3} ; \delta\right):= \\
1+2 y_{3} H\left(y_{\alpha}\right)+2 \delta \frac{\operatorname{Re}\left\langle\mathbf{h}_{0}\left(y_{\alpha}\right), \mathbf{h}_{1}\left(y_{\alpha}\right)\right\rangle}{\left|\mathbf{h}_{0}\left(y_{\alpha}\right)\right|^{2}}+\mathcal{O}\left(\left(\delta+y_{3}\right)^{2}\right) .
\end{array}\right.
$$

Relying on this formula, one can exhibit the asymptotic behavior of the skin depth $\mathcal{L}\left(\sigma, y_{\alpha}\right)$ for high conductivity $\sigma$, cf. [4, Th. 4.2]:

Theorem 3.2. Let $\Sigma$ be a regular surface with mean curvature $H$. Recall that $\ell(\sigma)$ is defined by (3.10). We assume that $\mathbf{h}_{0}\left(y_{\alpha}\right) \neq 0$. The skin depth $\mathcal{L}\left(\sigma, y_{\alpha}\right)$ has the following behavior for high conductivity:

$$
\mathcal{L}\left(\sigma, y_{\alpha}\right)=\ell(\sigma)\left(1+H\left(y_{\alpha}\right) \ell(\sigma)+\mathcal{O}\left(\sigma^{-1}\right)\right), \quad \sigma \rightarrow \infty
$$

Remark 3.3. The higher order terms $\mathcal{O}\left(\sigma^{-1}\right)$ in equation (3.13) do depend on the data $\mathbf{j}$ of problem (2.1).

\section{AXISYMMETRIC DOMAINS}

In order to perform scalar two dimensional computations which could represent correctly the features of a three-dimensional problem, we choose to consider an axisymmetric configuration in which $\Omega_{+}$and $\Omega_{-}$are axisymmetric domains with the same axis $\Xi_{0}$ : 
in cylindrical coordinates $(r, \theta, z)$ associated with this axis, there exists bi-dimensional "meridian" domains $\Omega^{\mathrm{m}}$ and $\Omega_{ \pm}^{\mathrm{m}}$ such that

$$
\begin{gathered}
\Omega=\left\{\mathbf{x} \in \mathbb{R}^{3} \mid(r, z) \in \Omega^{\mathrm{m}}, \theta \in \mathbb{T}\right\} \\
\Omega_{ \pm}=\left\{\mathbf{x} \in \mathbb{R}^{3} \mid(r, z) \in \Omega_{ \pm}^{\mathrm{m}}, \theta \in \mathbb{T}\right\} .
\end{gathered}
$$

Here $\mathbb{T}=\mathbb{R} /(2 \pi \mathbb{Z})$ is the one-dimensional torus. We denote by $\Gamma^{\mathrm{m}}$ and $\Sigma^{\mathrm{m}}$ the meridian curves corresponding to $\Gamma$ and $\Sigma$, respectively, and by $\Gamma_{0}, \Gamma_{0}^{+}$the following subsets of the rotation axis $\Xi_{0}$, see Fig. 3

$$
\Gamma_{0}=\Xi_{0} \cap \bar{\Omega}^{\mathrm{m}} \quad \text { and } \quad \Gamma_{0}^{+}=\Xi_{0} \cap \bar{\Omega}_{+}^{\mathrm{m}}
$$

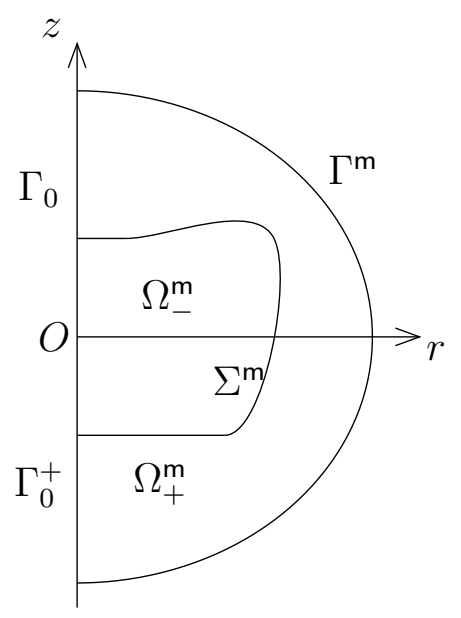

FIGURE 3. The meridian domain $\Omega^{\mathrm{m}}=\Omega_{-}^{\mathrm{m}} \cup \Omega_{+}^{\mathrm{m}} \cup \Sigma^{\mathrm{m}}$ with boundary $\partial \Omega^{\mathrm{m}}=\Gamma^{\mathrm{m}} \cup \Gamma_{0}$

On such an axisymmetric configuration we consider a modification of problem (2.1)(2.2): We take $\mathbf{j} \equiv 0$ and impose instead of (2.2) non-homogeneous magnetic boundary conditions

$$
\mathbf{E} \cdot \mathbf{n}=0 \quad \text { and } \quad \mathbf{H} \times \mathbf{n}=\mathbf{G} \times \mathbf{n} \quad \text { on } \Gamma,
$$

for a given data $\mathbf{G} \in \mathbf{H}(\operatorname{curl}, \Omega):=\left\{\mathbf{u} \in \mathbf{L}^{2}(\Omega) \mid \operatorname{curl} \mathbf{u} \in \mathbf{L}^{2}(\Omega)\right\}$. Then, the variational problem for the magnetic field $\mathbf{H}_{(\delta)}$ solution of the Maxwell equations (2.1)-(4.1) writes

Find $\mathbf{H}_{(\delta)} \in \mathbf{H}_{0}(\operatorname{curl}, \Omega)+\mathbf{G}$, such that for all $\mathbf{K} \in \mathbf{H}_{0}(\operatorname{curl}, \Omega)$,

$$
\int_{\Omega}\left(\frac{1}{\varepsilon(\delta)} \operatorname{curl} \mathbf{H}_{(\delta)} \cdot \operatorname{curl} \mathbf{K}-\kappa^{2} \mathbf{H}_{(\delta)} \cdot \mathbf{K}\right) \mathrm{d} \mathbf{x}=0 .
$$


4.1. Formulation in cylindrical components. For a vector field $\mathbf{H}=\left(H_{1}, H_{2}, H_{3}\right)$, we introduce its cylindrical components $\left(H_{r}, H_{\theta}, H_{z}\right)$ according to

$$
\left\{\begin{array}{l}
H_{r}(r, \theta, z)=H_{1}(\mathbf{x}) \cos \theta+H_{2}(\mathbf{x}) \sin \theta, \\
H_{\theta}(r, \theta, z)=-H_{1}(\mathbf{x}) \sin \theta+H_{2}(\mathbf{x}) \cos \theta, \\
H_{z}(r, \theta, z)=H_{3}(\mathbf{x}),
\end{array}\right.
$$

and we set

$$
\breve{\mathbf{H}}(r, \theta, z)=\left(H_{r}(r, \theta, z), H_{\theta}(r, \theta, z), H_{z}(r, \theta, z)\right) .
$$

We say that $\mathbf{H}$ is axisymmetric if $\mathbf{H}$ does not depend on the angular variable $\theta$.

The Maxwell problem (4.2) is axisymmetric, which means that, expressed in cylindrical variables $(r, \theta, z)$ and components $\breve{\mathbf{H}}$, its coefficients do not depend on $\theta[9,1,15]$. Recall that for a vector field $\mathbf{H}=\left(H_{1}, H_{2}, H_{3}\right)$ the cylindrical components of its curl write

$$
\left\{\begin{array}{l}
(\operatorname{curl} \mathbf{H})_{r}=\frac{1}{r} \partial_{\theta} H_{z}-\partial_{z} H_{\theta}, \\
(\operatorname{curl} \mathbf{H})_{\theta}=\partial_{z} H_{r}-\partial_{r} H_{z}, \\
(\operatorname{curl} \mathbf{H})_{z}=\frac{1}{r}\left(\partial_{r}\left(r H_{\theta}\right)-\partial_{\theta} H_{r}\right) .
\end{array}\right.
$$

As a consequence, if the right-hand side $\mathbf{G}$ is axisymmetric, and if (4.2) has a unique solution, then this solution is axisymmetric. According to (4.3), when $\mathbf{H}$ is axisymmetric its curl reduces to

$$
\left\{\begin{array}{l}
(\operatorname{curl} \mathbf{H})_{r}=-\partial_{z} H_{\theta}, \\
(\operatorname{curl} \mathbf{H})_{\theta}=\partial_{z} H_{r}-\partial_{r} H_{z}, \\
(\operatorname{curl} \mathbf{H})_{z}=\frac{1}{r} \partial_{r}\left(r H_{\theta}\right) .
\end{array}\right.
$$

4.2. Axisymmetric orthoradial problem. We say that $\mathbf{H}$ is orthoradial if its components $H_{r}$ and $H_{z}$ are zero.

We assume that $\mathbf{G}$ is axisymmetric and orthoradial, i.e.,

$$
\breve{\mathbf{G}}(r, \theta, z)=(0, \mathrm{~g}(r, z), 0) .
$$

The components $\left(H_{r}, H_{z}\right)$ and $H_{\theta}$ being uncoupled in (4.4), and the solution of problem (4.2) being unique, we obtain that this solution is orthoradial

$$
\breve{\mathbf{H}}_{(\delta)}(r, \theta, z)=\left(0, \mathrm{~h}_{(\delta)}(r, z), 0\right) .
$$

4.2.1. Variational formulation. In this framework, the change from Cartesian to cylindrical coordinates requires the modification of the solution spaces $\mathbf{H}_{0}(\operatorname{curl}, \Omega)$ used in problem (4.2). Precisely, the weighted space characterizing the orthoradial component $\mathrm{h}_{(\delta)}(r, z)$ is

$$
\mathrm{V}_{1, \Gamma^{\mathrm{m}}}^{1}\left(\Omega^{\mathrm{m}}\right)=\left\{v \in \mathrm{H}_{1}^{1}\left(\Omega^{\mathrm{m}}\right) \mid v \in \mathrm{L}_{-1}^{2}\left(\Omega^{\mathrm{m}}\right) \quad \text { and } \quad v=0 \quad \text { on } \quad \Gamma^{\mathrm{m}}\right\} .
$$

Here,

$$
\mathrm{H}_{1}^{1}\left(\Omega^{\mathrm{m}}\right)=\left\{v \in \mathrm{L}_{1}^{2}\left(\Omega^{\mathrm{m}}\right) \mid \partial_{r}^{j} \partial_{z}^{1-j} v \in \mathrm{L}_{1}^{2}\left(\Omega^{\mathrm{m}}\right), j=0,1\right\}
$$


and for all $\alpha \in \mathbb{R}$, the space $\mathrm{L}_{\alpha}^{2}\left(\Omega^{\mathrm{m}}\right)$ is the set of measurable functions $v(r, z)$ such that

$$
\|v\|_{\mathrm{L}_{\alpha}^{2}\left(\Omega^{\mathrm{m}}\right)}^{2}=\int_{\Omega^{\mathrm{m}}}|v|^{2} r^{\alpha} d r d z<+\infty .
$$

Remark 4.1. The space $\mathrm{V}_{1, \Gamma^{\mathrm{m}}}^{1}\left(\Omega^{\mathrm{m}}\right)$ incorporates essential boundary conditions, in particular on $\Gamma_{0}$, where $v=0$, see [1, Remark II.1.1].

This leads us to solve the following two-dimensional scalar problem set in $\Omega^{\mathrm{m}}$.

Find $\mathrm{h}_{(\delta)} \in \mathrm{V}_{1, \Gamma^{\mathrm{m}}}^{1}\left(\Omega^{\mathrm{m}}\right)+\mathrm{g}$ such that for all $\mathrm{w} \in \mathrm{V}_{1, \Gamma^{\mathrm{m}}}^{1}\left(\Omega^{\mathrm{m}}\right)$,

$$
a^{\delta}\left(\mathrm{h}_{(\delta)}, \mathrm{w}\right)=0,
$$

where

$$
a^{\delta}(\mathrm{h}, \mathrm{w}):=\int_{\Omega^{\mathrm{m}}} \frac{1}{\varepsilon(\delta)}\left(\partial_{z} \mathrm{~h} \partial_{z} \mathrm{w}+\frac{1}{r} \partial_{r}(r \mathrm{~h}) \frac{1}{r} \partial_{r}(r \mathrm{w})\right) r d r d z-\kappa^{2} \int_{\Omega^{\mathrm{m}}} \mathrm{h} \mathrm{w} r d r d z .
$$

4.2.2. Asymptotic expansion. Let $(r(\xi), z(\xi))=\boldsymbol{\tau}(\xi), \xi \in(0, L)$, be an arc-length coordinate on the interface $\Sigma^{\mathrm{m}}$. Here $\xi \mapsto \tau(\xi)$ is a $\mathcal{C}^{\infty}$ function, and $L$ is the length of the curve $\Sigma^{\mathrm{m}}$. Let $\left(\xi, y_{3}\right)$ be the associate normal coordinate system in a tubular neighborhood of $\Sigma^{\mathrm{m}}$ inside $\Omega_{-}^{\mathrm{m}}$. Then the normal vector $\mathbf{n}(\xi)$ at the point $\boldsymbol{\tau}(\xi)$ can be written as (Frenet frame)

$$
\mathbf{n}(\xi)=\left(-z^{\prime}(\xi), r^{\prime}(\xi)\right) \quad \text { with } \quad z^{\prime}(\xi)=\frac{d z}{d \xi} \quad \text { and } \quad r^{\prime}(\xi)=\frac{d r}{d \xi}
$$

Finally we denote by $k(\xi)$ the curvature of $\Sigma^{\mathrm{m}}$ in $\boldsymbol{\tau}(\xi)$.

In accordance with (3.1)-(3.2)-(3.3), we can exhibit series expansions in powers of $\delta$ for the magnetic field $\mathrm{h}_{(\delta)}$ which we denote by $\mathrm{h}_{(\delta)}^{+}$in the dielectric part $\Omega_{+}$, and by $\mathrm{h}_{(\delta)}^{-}$ in the conducting part $\Omega_{-}$:

$$
\begin{gathered}
\mathrm{h}_{(\delta)}^{+}(r, z)=\mathrm{h}_{0}^{+}(r, z)+\delta \mathrm{h}_{1}^{+}(r, z)+\mathcal{O}\left(\delta^{2}\right) \\
\mathrm{h}_{(\delta)}^{-}(r, z)=\mathrm{h}_{0}^{-}(r, z ; \delta)+\delta \mathrm{h}_{1}^{-}(r, z ; \delta)+\mathcal{O}\left(\delta^{2}\right), \quad \mathrm{h}_{j}^{-}(r, z ; \delta)=\chi\left(y_{3}\right) \mathfrak{h}_{j}^{\theta}\left(\xi, \frac{y_{3}}{\delta}\right) .
\end{gathered}
$$

Here the profiles $\mathfrak{h}_{j}^{\theta}$ are defined on $\Sigma^{\mathrm{m}} \times \mathbb{R}^{+}$. Hereafter, we focus on the first terms $\mathrm{h}_{0}^{+}$, $\mathfrak{h}_{0}^{\theta}, \mathrm{h}_{1}^{+}$, and $\mathfrak{h}_{1}^{\theta}$. We introduce the interior and boundary operators

$$
\begin{aligned}
& \mathrm{D}\left(r, z ; \partial_{r}, \partial_{z}\right)=\partial_{r}^{2}+\frac{1}{r} \partial_{r}+\partial_{z}^{2}-\frac{1}{r^{2}} \\
& \quad \text { and } \mathrm{B}\left(\xi ; \partial_{r}, \partial_{z}\right)=-z^{\prime}(\xi)\left(\partial_{r}+\frac{1}{r}\right)+r^{\prime}(\xi) \partial_{z} .
\end{aligned}
$$

In appendix B.1, we give the expansion of these operators in power series of $\delta$ inside the domain $\Omega_{-}$and on the interface $\Sigma^{\mathrm{m}}$. The terms $\mathrm{h}_{0}^{+}, \mathfrak{h}_{0}^{\theta}, \mathrm{h}_{1}^{+}$, and $\mathfrak{h}_{1}^{\theta}$ satisfy the following problems coupled by their boundary conditions on the interface $\Sigma^{\mathrm{m}}$ (corresponding to 
$Y=0)$ - compare with (3.4)-(3.5), (3.8)-(3.9):

$$
\begin{gathered}
\left\{\begin{array}{lll}
\mathrm{Dh}_{0}^{+}+\kappa^{2} \mathrm{~h}_{0}^{+} & =0 \text { in } \Omega_{+}^{\mathrm{m}}, \\
\mathrm{Bh}_{0}^{+} & =0 \text { on } \Sigma^{\mathrm{m}}, \\
\mathrm{h}_{0}^{+} & =\mathrm{g} \text { on } \Gamma^{\mathrm{m}} \cup \Gamma_{0}^{+},
\end{array}\right. \\
\begin{cases}\left(\partial_{Y}^{2}-\lambda^{2}\right) \mathfrak{h}_{0}^{\theta}= & 0 \text { for } 0<Y<+\infty, \\
\mathfrak{h}_{0}^{\theta}= & \mathrm{h}_{0}^{+} \text {for } Y=0,\end{cases}
\end{gathered}
$$

with $\lambda$ defined by (3.5), from which we deduce:

$$
\mathfrak{h}_{0}^{\theta}(\xi, Y)=\mathrm{e}^{-\lambda Y} \mathrm{~h}_{0}^{+}(\boldsymbol{\tau}(\xi)) .
$$

The next problem in the dielectric part is

$$
\left\{\begin{array}{lll}
\mathrm{Dh}_{1}^{+}+\kappa^{2} \mathrm{~h}_{1}^{+} & =0 & \text { in } \Omega_{+}^{\mathrm{m}}, \\
\mathrm{Bh}_{1}^{+} & =-i \partial_{Y} \mathfrak{h}_{0}^{\theta} & \text { on } \Sigma^{\mathrm{m}}, \\
\mathrm{h}_{1}^{+} & =0 & \text { on } \Gamma^{\mathrm{m}} \cup \Gamma_{0}^{+},
\end{array}\right.
$$

Thus (4.10) yields that $\mathrm{h}_{1}^{+}$satisfies $\mathrm{Bh}_{1}^{+}=\left.i \lambda \mathrm{h}_{0}^{+}\right|_{\Sigma^{\mathrm{m}}}$ on the interface.

The next problem in the conductor part is

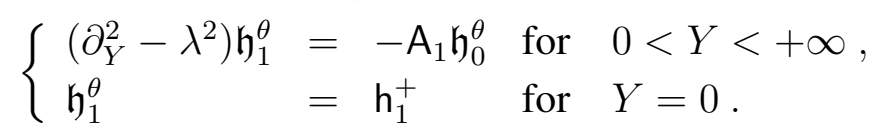

Here, $\mathrm{A}_{1} \mathfrak{h}_{0}^{\theta}=-\left(k+\frac{z^{\prime}}{r}\right)(\xi) \partial_{Y} \mathfrak{h}_{0}^{\theta}$. From (4.10), we infer $\mathrm{A}_{1} \mathfrak{h}_{0}^{\theta}=\left.\mathrm{e}^{-\lambda Y} \lambda\left(k+\frac{z^{\prime}}{r}\right)(\xi) \mathrm{h}_{0}^{+}\right|_{\Sigma^{\mathrm{m}}}$. Then, from equation (4.12), we obtain

$$
\mathfrak{h}_{1}^{\theta}(\xi, Y)=\mathrm{e}^{-\lambda Y}\left[\mathrm{~h}_{1}^{+}(\boldsymbol{\tau}(\xi))+\frac{Y}{2}\left(k+\frac{z^{\prime}}{r}\right)(\xi) \mathbf{h}_{0}^{+}(\boldsymbol{\tau}(\xi))\right] .
$$

Note that we can also deduce this profile $\mathfrak{h}_{1}^{\theta}$ from equations (3.5) and (3.9), but this is not obvious because the cylindrical coordinates are not a normal coordinate system, see Appendix B.2.

Remark 4.2. Subsequently, we assume that the data $g$ is a real valued function. Thus, the right hand side of the boundary value problem (4.8) is real. Hence $h_{0}^{+}$is a real valued function. Recall that $\mathrm{Bh}_{1}^{+}=\left.\kappa \mathrm{e}^{i \pi / 4} \mathrm{~h}_{0}^{+}\right|_{\Sigma}$. From the boundary value problem (4.11), we infer: $\operatorname{Re}_{1}^{+}=\operatorname{Im~h}_{1}^{+}$. We will exploit this relationship in the numerical simulations of skin effect, see $\S 6$.

4.3. Configurations chosen for computations. We consider three classes of geometric configurations: one cylindrical configuration (A) and two spheroidal configurations (B and $\mathrm{C}$ ).

Configuration A: cylindrical geometry. We assume that $\Omega$ is a circular cylinder of radius $r_{1}$ and length $\ell_{1}$, and $\Omega_{-}$is a coaxial cylinder of radius $r_{0}$ and length $\ell_{0}$. Hence, $\Omega^{\mathrm{m}}$ is a rectangle of width $r_{1}$ and length $\ell_{1}$, and $\Omega_{-}^{\mathrm{m}}$ is a coaxial rectangle of width $r_{0}$ and length $\ell_{0}$, see Figure 4 . We choose the parameters $r_{0}=1, \ell_{0}=2, r_{1}=2, \ell_{1}=4$ in computations. 


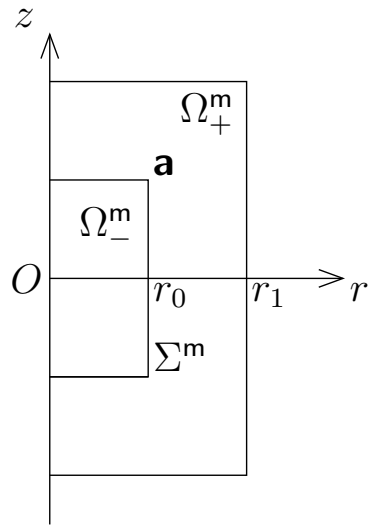

FIGURE 4. The meridian domain $\Omega^{\mathrm{m}}$ in configuration $\mathrm{A}$

Configuration B: spheroidal geometry. We assume that $\Omega$ is a spheroid, and $\Omega_{-}$is a coaxial spheroid. We denote by $a$ and $c$, respectively $b$ and $d$, the semimajor and semiminor axis of $\Omega_{-}$, respectively $\Omega$. Hence, $\Omega^{\mathrm{m}}$ is a semi-ellipse with semimajor axis $b$ and semiminor axis $d$, and $\Omega_{-}^{m}$ is a coaxial semi-ellipse with axis lengths $a$ and $c$, see Figure 5. In computations, we consider the following parameters

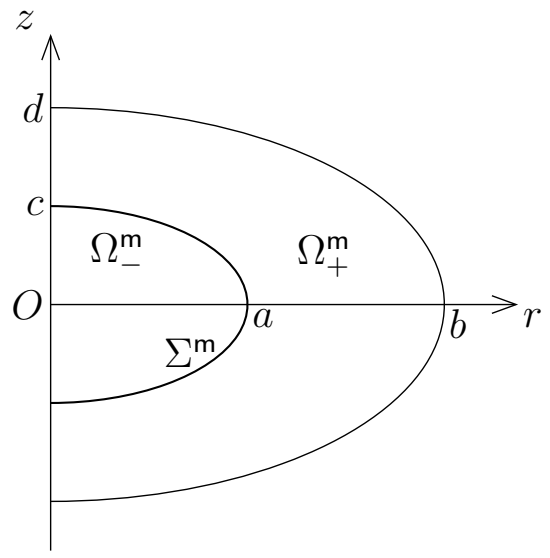

FIGURE 5. The meridian domain $\Omega^{\mathrm{m}}$ in configuration $\mathrm{B} 1$

Configuration B1: $\quad a=2, b=4, c=1, d=2, \quad$ (oblate spheroid)

Configuration B2: $a=4, b=8, c=1, d=2$, (more oblate spheroid).

Note that all the numerical parameters are given in SI units, in particular, $a, b, c$, and $d$ are defined in meters, and the conductivity $\sigma$ is defined in siemens per meter $S . m^{-1}$.

Configuration $C$ : spheroidal geometry. We introduce a configuration $\mathrm{C}$, switching the roles of the subdomains $\Omega_{-}^{\mathrm{m}}$ and $\Omega_{+}^{\mathrm{m}}$ in the configuration $\mathrm{B}$, see Figure 6 . We denote by 


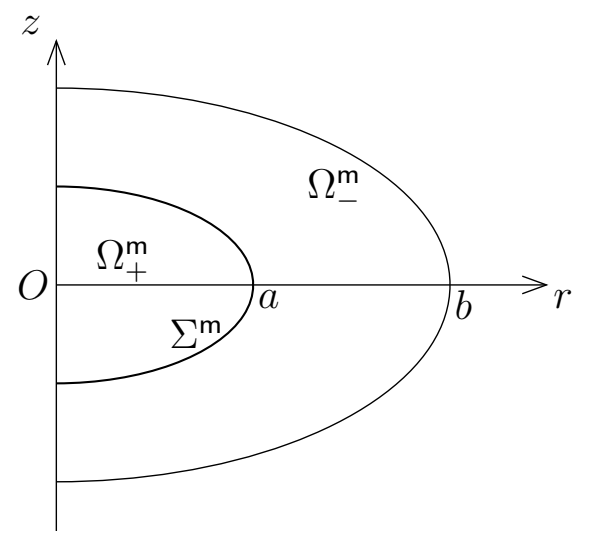

FIGURE 6. The meridian domain $\Omega^{\mathrm{m}}$ in configuration $\mathrm{C} 1$

$\mathrm{C} 1$ and $\mathrm{C} 2$ the configuration $\mathrm{C}$, which correspond to the choice of parameters $a, b, c, d$, see (4.14).

Right hand sides of problems. In configurations $\mathrm{A}$ and $\mathrm{B}$, we take the data $\mathrm{g}=r$. Hence, $\mathrm{h}_{(\delta)}$ satisfies the following inhomogeneous Dirichlet boundary condition

$$
\mathrm{h}_{(\delta)}(r, z)=r \quad \text { on } \quad \Gamma^{\mathrm{m}} .
$$

For configuration $\mathrm{C} 1$, we take $\mathrm{g}=0$ and an interior data $f:=(\operatorname{curl} \mathbf{j})_{\theta}$ with support inside $\Omega_{+}^{\mathrm{m}}: f=10^{2}$ if $r^{2} / 4+z^{2} \leqslant 0.8$ and $f=0$ otherwise.

\section{FINITE ELEMENT DISCRETIZATIONS AND COMPUTATIONS}

5.1. Finite element method. In this section, we consider two benchmarks for the computational domain: configuration A, see Figure 4, and configuration B, see Figure 5. We use high order elements available in the finite element library Mélina, see [13], and quadrangular meshes in the meridian domain. We discretize the variational problem (4.5). The script used to solve the problem (4.5) is adapted from [2]. In the computations, we fix the angular frequency $\omega=3.10^{7}$. We denote by $\mathrm{h}_{(\delta)}^{p, \mathfrak{M}}$ the computed solution of the discretized problem (4.5) with an interpolation degree $p$ and a mesh $\mathfrak{M}$. We define

$$
A_{\sigma}^{p, \mathfrak{M}}:=\left\|\mathrm{h}_{(\delta)}^{p, \mathfrak{M}}\right\|_{\mathrm{L}_{1}^{2}\left(\Omega_{-}^{\mathrm{m}}\right)} \quad \text { with } \quad \sigma=\omega \varepsilon_{0} \delta^{-2} \text {, cf. (2.5) . }
$$

5.2. Interpolation degree. We first check the convergence when the interpolation degree of the finite elements increases.

5.2.1. Configuration $B 1$. We consider the discretized problem with different degrees: $Q_{p}{ }^{2}$, for all $p=1, \cdots, 20$, and with three different meshes $\mathfrak{M}_{1}, \mathfrak{M}_{3}$ and $\mathfrak{M}_{6}$ with 1,3 or 6 layers of elongated elements in the skin region of the conductor $\Omega_{-}^{\mathrm{m}}$, see Figure 7 .

\footnotetext{
${ }^{2}$ Recall that $Q_{p}$ is the vector space of polynomials of two variables and partial degree $p$ defined on the reference element $\widehat{K}:=[0,1] \times[0,1]$
} 

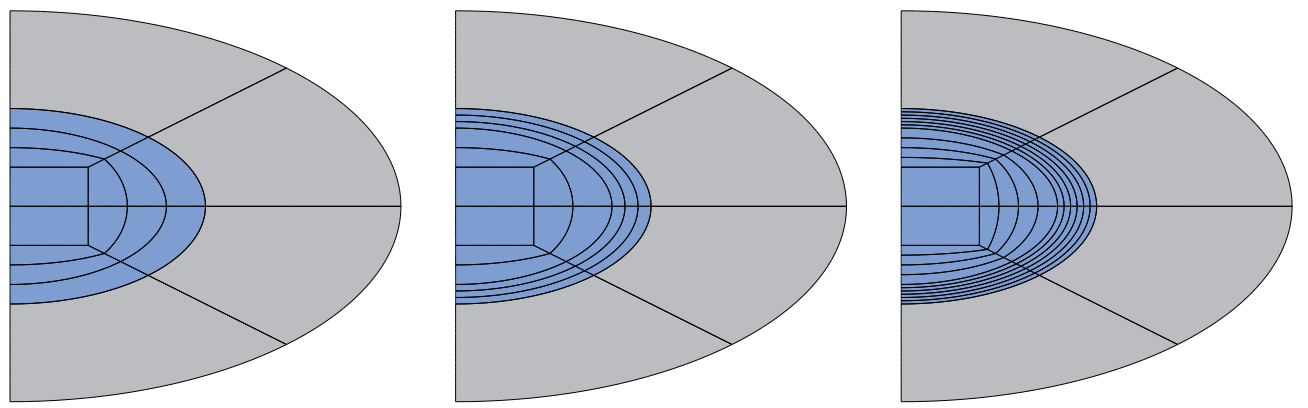

FIgURE 7. The meshes $\mathfrak{M}_{1}, \mathfrak{M}_{3}$ and $\mathfrak{M}_{6}$ for configuration $\mathrm{B} 1$

We represent in Figure 8 the absolute value of the difference between the weighted norms $A_{\sigma}^{p, \mathfrak{M}_{1}}$ and $A_{\sigma}^{20, \mathfrak{M}_{6}}$, versus $p$ in semilogarithmic coordinates, and in each case: $\sigma=5$ with circles, $\sigma=20$ with squares, and $\sigma=80$ with diamonds. We refer to [19] for theoretical results of convergence for the $p$-version in presence of an exponentially decreasing boundary layer. In Figure 9 we use mesh $\mathfrak{M}_{3}$ instead of $\mathfrak{M}_{1}$.

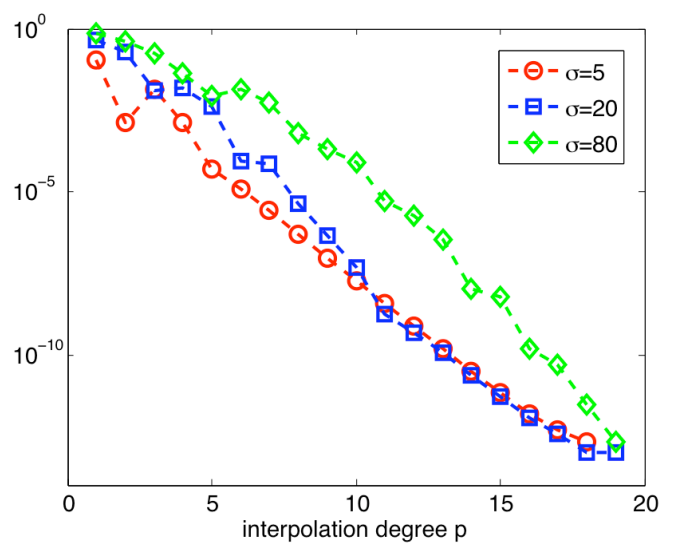

FIGURE 8. Graph of $\left|A_{\sigma}^{p, \mathfrak{M}_{1}}-A_{\sigma}^{20, \mathfrak{M}_{6}}\right|$ with respect to $p=1, \cdots, 19$ in semi- $\log$ coordinates, for $\sigma \in\{5,20,80\}$ and configuration B1

In Figure 10, we plot in log-log coordinates the weighted norm $A_{\sigma}^{16, \mathfrak{M}_{3}}$ with respect to $\sigma=5,20,80,100,200,300,400$ with circles, and the graph of $\sigma \mapsto \sigma^{-1 / 4}$ by a solid line. The figure shows that $A_{\sigma}^{16, \mathfrak{M}_{3}}$ behaves like $\sigma^{-1 / 4}$ when $\sigma \rightarrow \infty$. This behavior is consistent with the asymptotic expansion (3.2) and the estimate (3.6), (recall formula $(2.5))$. 


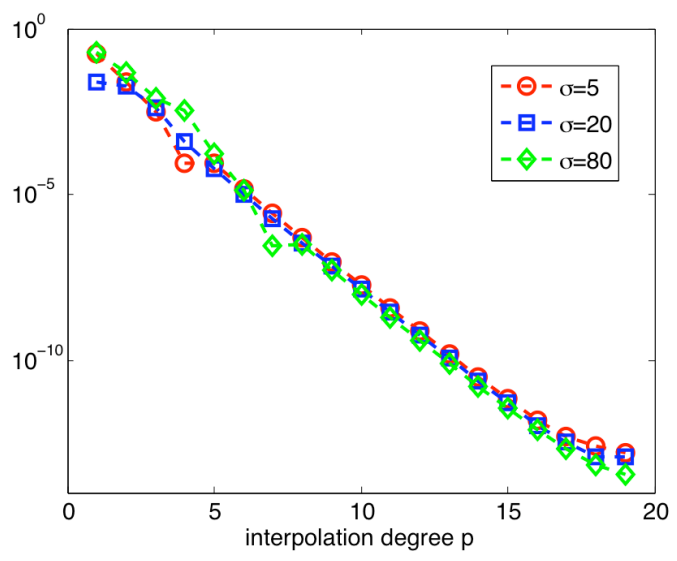

FIGURE 9. Graph of $\left|A_{\sigma}^{p, \mathfrak{M}_{3}}-A_{\sigma}^{20, \mathfrak{M}_{6}}\right|$ with respect to $p=1, \cdots, 19$ in semi- $\log$ coordinates, for $\sigma \in\{5,20,80\}$ and configuration B1

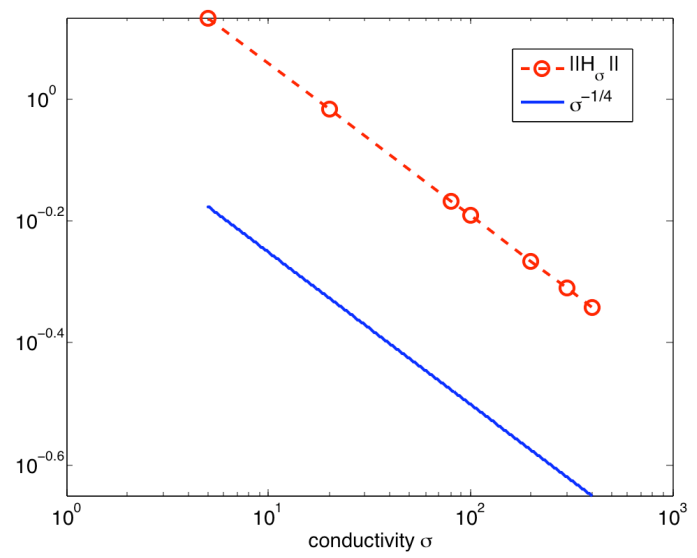

FIGURE 10. In circle: $A_{\sigma}^{16, \mathfrak{M}_{3}}$ for $\sigma=5,20,80,100,200,300,400$. In solid line: the graph of the function $\sigma \mapsto \sigma^{-1 / 4}$ in log-log coordinates for configuration B1 
5.2.2. Configuration A. We consider a family of eight meshes with square elements $\mathfrak{M}_{k}, k=1, \ldots, 8$ with size $h=1 / k$, see Figure 11 . We represent in Figure 12 the
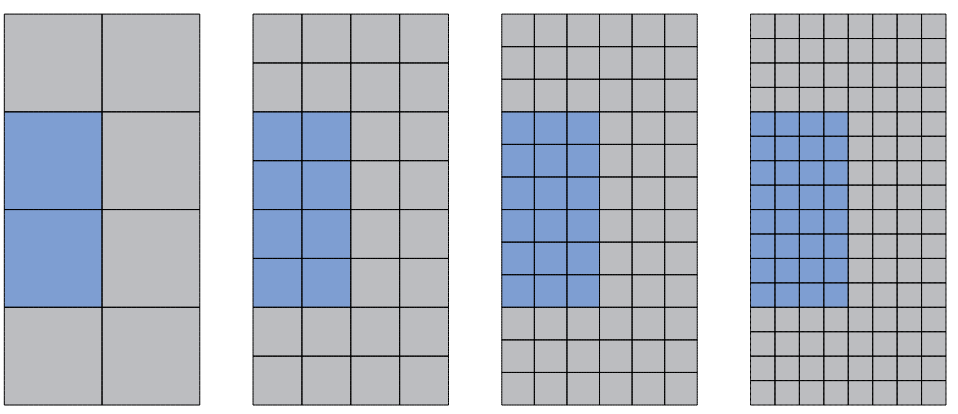

FIgURE 11. Meshes $\mathfrak{M}_{1}, \mathfrak{M}_{2}, \mathfrak{M}_{3}$, and $\mathfrak{M}_{4}$ for configuration A

absolute value of the difference between $A_{\sigma}^{p, \mathfrak{M}_{2}}$ and $A_{\sigma}^{16, \mathfrak{M}_{3}}$, versus $p$ in semilogarithmic coordinates, and in each case: $\sigma=5$ with circles, $\sigma=20$ with squares, and $\sigma=80$ with diamonds. The figure shows that $A_{\sigma}^{p, \mathfrak{M}_{2}}$ approximates $A_{\sigma}^{16, \mathfrak{M}_{3}}$ better than $10^{-4}$ when $p \geqslant 12$.

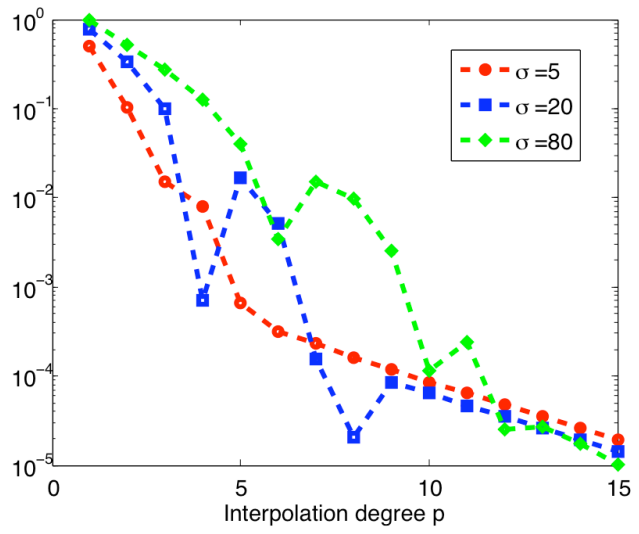

FIGURE 12. Graph of $\left|A_{\sigma}^{p, \mathfrak{M}_{2}}-A_{\sigma}^{16, \mathfrak{M}_{3}}\right|$ with respect to $p=1, \cdots, 15$ in semi-log coordinates, for $\sigma \in\{5,20,80\}$ for configuration A 
5.3. Stability of the h-version. In this subsection, we check the convergence of the discretized problem in configuration A for several meshes $\mathfrak{M}_{k}$ of the computational domain when $k$ increases. We fix the interpolation degree $p=2$ of the finite elements and use the square meshes $\mathfrak{M}_{k}$. We plot in Figure 13 the absolute value of the difference between $A_{\sigma}^{2, \mathfrak{M}_{k}}$ and $A_{\sigma}^{6, \mathfrak{M}_{8}}$ with respect to $k=1, . ., 8$, in log-log coordinates.

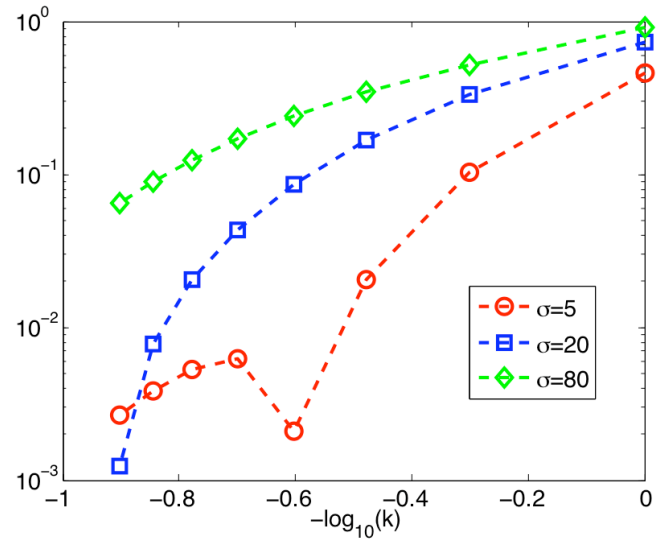

FIGURE 13. Graph of $\left|A_{\sigma}^{2, \mathfrak{M}_{k}}-A_{\sigma}^{6, \mathfrak{M}_{8}}\right|$ with respect to $-\log _{10}(k)$ in semi-log coordinates, when $k=1, . ., 8$ and $\sigma=5,20,80$ for configuration A

\section{NUMERICAL SIMULATIONS OF SKIN EFFECT}

Recall that the asymptotic expansion of $\mathrm{h}_{(\delta)}$ is described in $\S 4.2 .2$. According to Remark 4.2 the first term $\mathrm{h}_{0}^{+}$is a real valued function. Hence the imaginary part of the magnetic field $\mathrm{h}_{(\delta)}$ is small in the dielectric $\Omega_{+}^{\mathrm{m}}$, because

$$
\left|\operatorname{Im~h}_{(\delta)}^{+}\right|=\mathcal{O}(\delta) \text {. }
$$

Thus, the imaginary part of the computed field is located in the conductor $\Omega_{-}^{\mathrm{m}}$. We display this imaginary part to highlight the boundary layer near the surface of the conductor, see Figures 15, 16 bottom, 17 and 18. In this section, $\tilde{\mathrm{h}}_{\sigma}:=\mathrm{h}_{(\delta)}^{p, \mathfrak{M}_{k}}$ denote the computed solution associated with the numerical parameters considered in each subsection.

6.1. Skin effect in configuration $\mathbf{A}$. We fix the mesh $\mathfrak{M}_{2}$, see Figure 11 , and the interpolation degree of the finite elements : $p=16$. Here $\tilde{\mathrm{h}}_{\sigma}=\mathrm{h}_{(\delta)}^{16, \mathfrak{M}_{2}}$ is computed in configuration A for several values of $\sigma$, see Figure 14. Similarly, we compute $\left|\operatorname{Im} \tilde{h}_{\sigma}\right|$, see Figure 15.

6.2. Skin effect in configuration B. Here $\tilde{\mathrm{h}}_{\sigma}=\mathrm{h}_{(\delta)}^{p, \mathfrak{M}_{3}}$ is the computed solution for several values of $\sigma$, with a fixed mesh $\mathfrak{M}_{3}$ (Figure 7). We represent $\left|\tilde{\mathrm{h}}_{\sigma}\right|$ and $\left|\operatorname{Im} \tilde{\mathrm{h}}_{\sigma}\right|$ in configuration $\mathrm{B} 1$, with an interpolation degree $p=16$, see Figure 

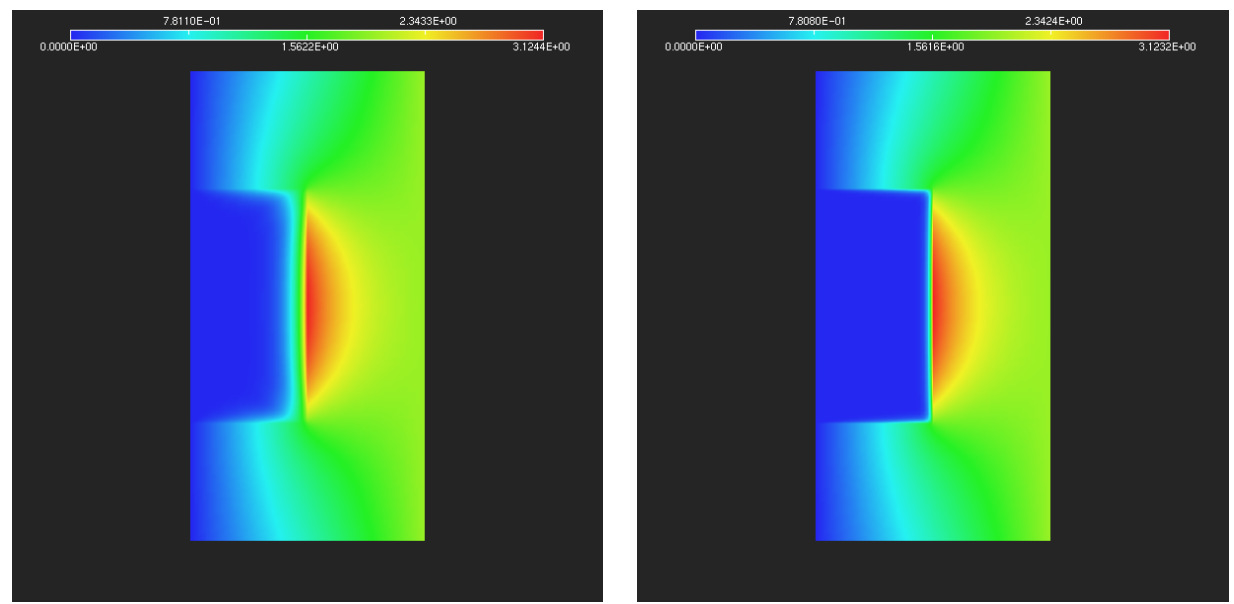

FIGURE 14. Configuration A. On the left, $\left|\tilde{\mathrm{h}}_{\sigma}\right|$ when $\sigma=5$. On the right, $\left|\tilde{\mathrm{h}}_{\sigma}\right|$ when $\sigma=80$
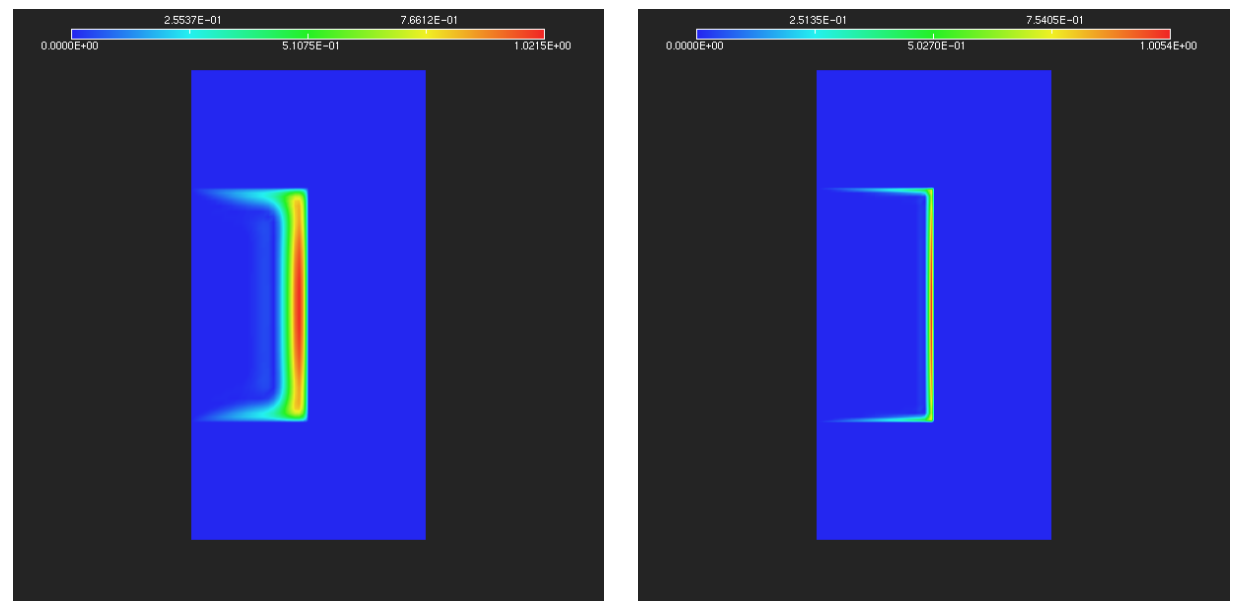

FIgure 15. Configuration A. On the left, $\left|\operatorname{Im} \tilde{\mathrm{h}}_{\sigma}\right|$ when $\sigma=5$. On the right, $\left|\operatorname{Im} \tilde{h}_{\sigma}\right|$ when $\sigma=80$

6.3. Skin effect in configurations $\mathbf{B}$ and $\mathbf{C}$. In order to exhibit the influence of the sign of the mean curvature $H$ of the interface $\Sigma$ on the skin effect, we fix in this subsection the conductivity $\sigma=5$. We perform computations in configurations B and C. We note that $H>0$ in configuration $\mathrm{B}$, and $H<0$ in configuration $\mathrm{C}$. We compute $\left|\operatorname{Im} \tilde{\mathrm{h}}_{\sigma}\right|$ in configurations B1 and C1, see Figure 17. We then compute $\left|\operatorname{Im} \tilde{h}_{\sigma}\right|$ in configurations B2 and $\mathrm{C} 2: H>0$, and $H<0$, see Figure 18 .

Figures 17 and 18 show that the skin depth is larger for a fixed conductivity when the mean curvature of the conducting body surface is larger. Moreover, the sign of the 

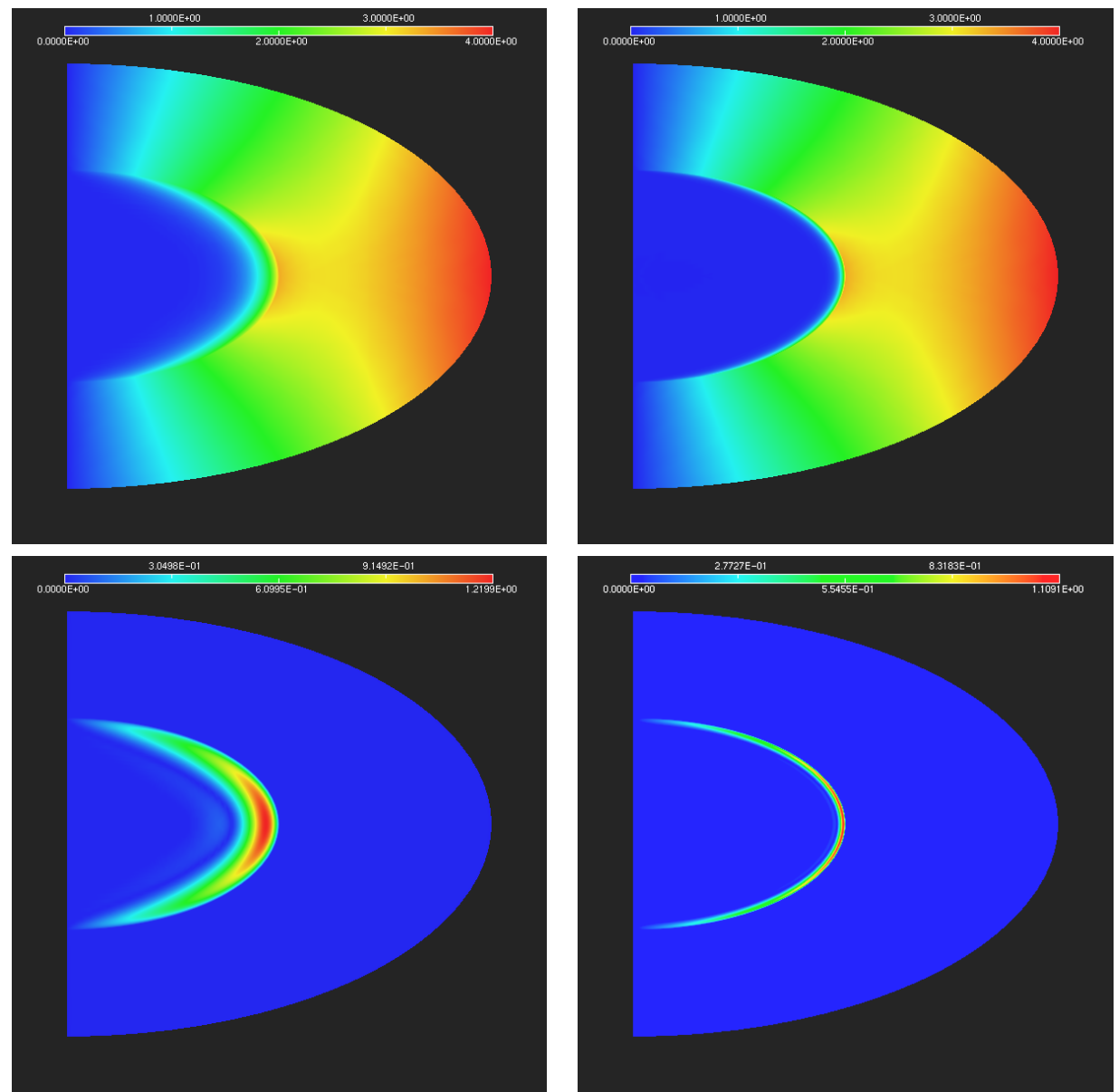

Figure 16. Configuration B1. At the top, $\left|\tilde{\mathrm{h}}_{\sigma}\right|$ when $\sigma=5$ (on the left), and $\sigma=80$ (on the right). At the bottom, $\left|\operatorname{Im} \tilde{\mathrm{h}}_{\sigma}\right|$ when $\sigma=5$ and $\sigma=80$

curvature has an influence on the skin depth. This length is larger in convex (Figures 17 and 18 on the left) than in concave conductors (same figures on the right). 

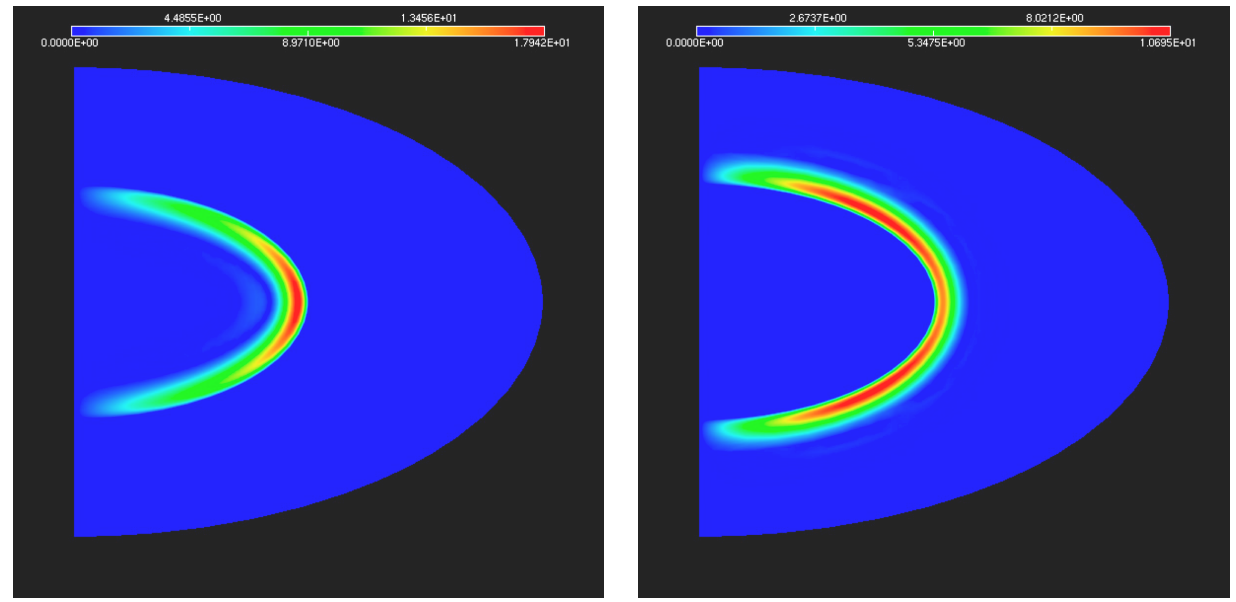

FIGURE 17. On the left, $\left|\operatorname{Im} \tilde{\mathrm{h}}_{\sigma}\right|$ in configuration B1 $(H>0)$. On the right, $\left|\operatorname{Im} \tilde{h}_{\sigma}\right|$ in configuration $\mathrm{C} 1(H<0) . \sigma=5$
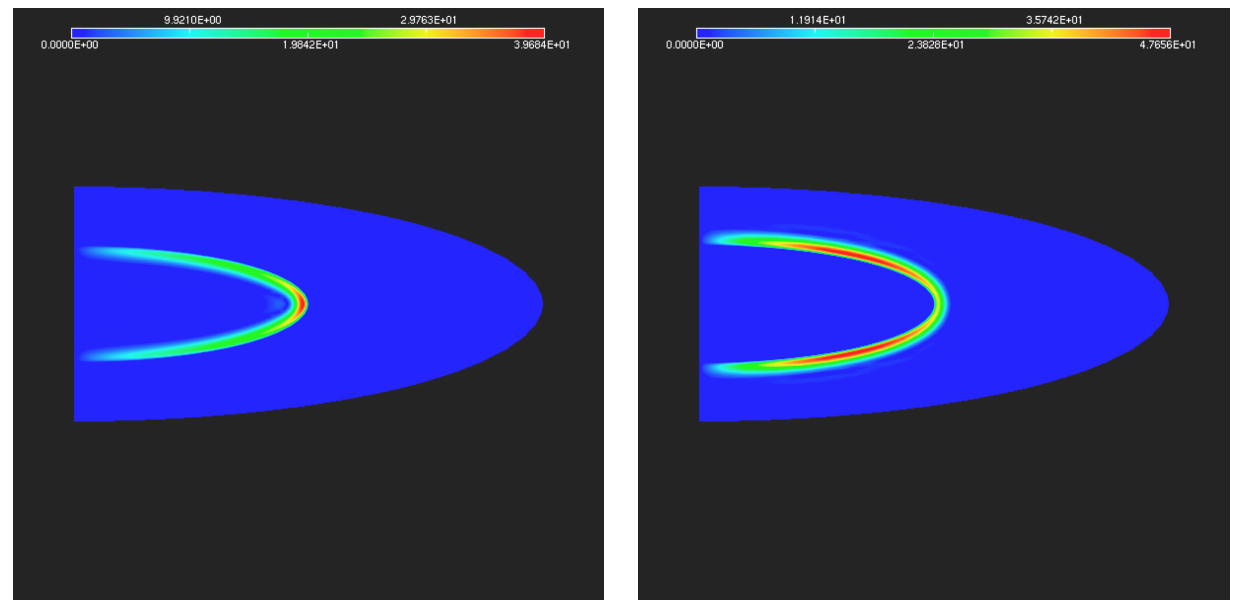

Figure 18. On the left, $\left|\operatorname{Im} \tilde{h}_{\sigma}\right|$ in configuration B2. On the right, $\left|\operatorname{Im} \tilde{\mathrm{h}}_{\sigma}\right|$ in configuration $\mathrm{C} 2 . \sigma=5$ 


\section{PostPRocessing}

In this section, we perform numerical treatments from computations in configuration B1, see subsection 6.2, and configuration A, see subsection 6.1, in order to investigate whether solutions are exponentially decreasing inside the conductor and with which rate. Let us recall that the standard skin depth $\ell(\sigma)$ is given by (3.10).

7.1. Configuration B. The mesh of the computational domain is the mesh $\mathfrak{M}_{3}$, see Figure 7. We extract values of $\left|\tilde{\mathrm{h}}_{\sigma}\right|$ in $\Omega_{-}^{\mathrm{m}}$ along edges of the mesh $\mathfrak{M}_{3}$ for $z=0$ : in this configuration, the normal coordinate writes $y_{3}:=2-r$.

Then, we perform a linear regression from values of $\log _{10}\left|\tilde{\mathrm{h}}_{\sigma}\left(y_{3}\right)\right|$ in the skin depth $\ell(\sigma)$, see Figure 19. We denote by $n(\sigma)$ the number of extracted values on the axis $z=0$ in the skin depth $\ell(\sigma)$. From the linear regression, we derive a numerical slope $\tilde{s}(\sigma)$ such that

$$
\log _{10}\left|\tilde{\mathrm{h}}_{\sigma}\left(y_{3}\right)\right|=-\tilde{s}(\sigma) y_{3}+b, \quad b \in \mathbb{R} .
$$
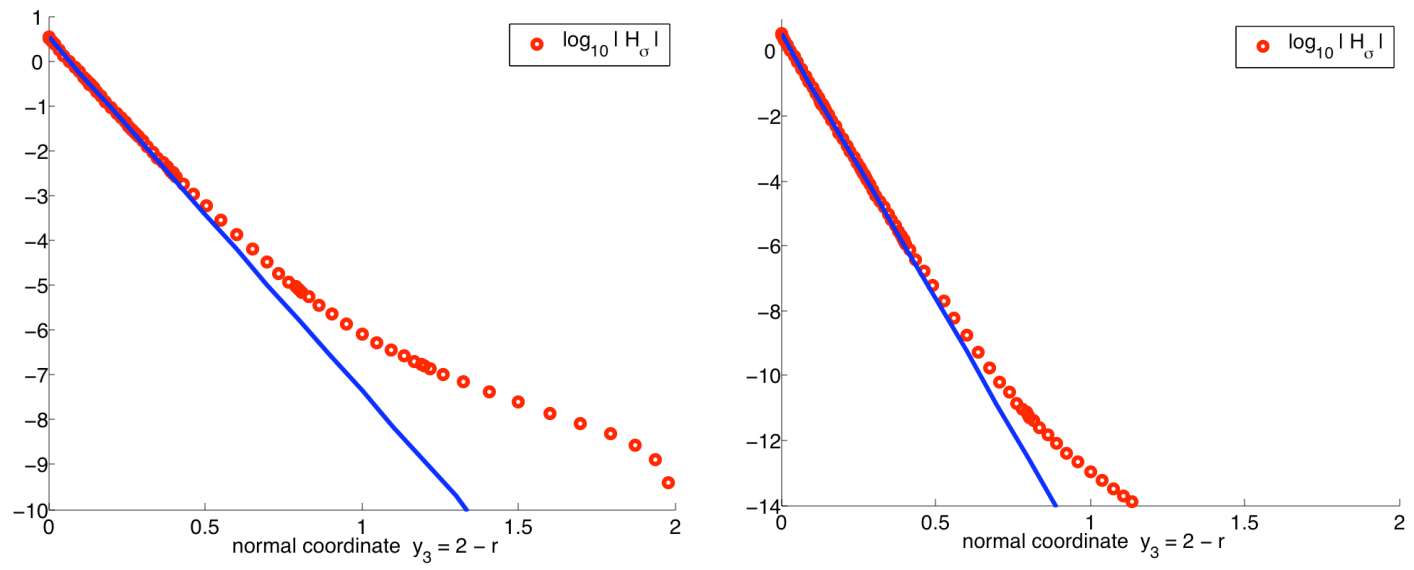

FIGURE 19. In circles, extracted values of $\log _{10}\left|\tilde{\mathrm{h}}_{\sigma}\left(y_{3}\right)\right|$. In solid line, linear regression of $\log _{10}\left|\tilde{\mathrm{h}}_{\sigma}\left(y_{3}\right)\right|$. On the left $\sigma=20$ (interpolation degree $p=12$ ). On the right, $\sigma=80$ (degree $p=16$ ). $n(20)=6$, $\tilde{s}(20)=7.86906$ and $n(80)=5, \tilde{s}(80)=16.29634$. Configuration B1

Accuracy of asymptotics. Relying on formula (3.12), we can derive a Taylor expansion of $\log _{10}\left|\underline{\mathfrak{T}}_{(\delta)}\left(y_{\alpha}, y_{3}\right)\right|$ with respect to $y_{3}$ : for $y_{3}$ small enough

$$
\begin{aligned}
\log _{10}\left|\underline{\mathfrak{T}}_{(\delta)}\left(y_{\alpha}, y_{3}\right)\right|=\log _{10}\left|\mathbf{h}_{0}\left(y_{\alpha}\right)\right| \\
\quad-s\left(y_{\alpha}, \sigma\right) y_{3}+\frac{\delta}{\ln 10}\left|\mathbf{h}_{0}\left(y_{\alpha}\right)\right|^{-2} \operatorname{Re}\left\langle\mathbf{h}_{0}\left(y_{\alpha}\right), \mathbf{h}_{1}\left(y_{\alpha}\right)\right\rangle+\mathcal{O}\left(\left(\delta+y_{3}\right)^{2}\right) .
\end{aligned}
$$

Here the function

$$
s\left(y_{\alpha}, \sigma\right):=\frac{1}{\ln 10}\left(\frac{1}{\ell(\sigma)}-H\left(y_{\alpha}\right)\right)
$$


depends on the skin depth $\ell(\sigma)$ and the mean curvature $H\left(y_{\alpha}\right)$ at the point $y_{\alpha}$ of the interface $\Sigma$. We note that the mean curvature of the surface $\Sigma$ is constant when $z=0$. Hence, we introduce hereafter the theoretical slope

$$
s(\sigma):=s\left(y_{\alpha}, \sigma\right) \text { when } \quad z=0 .
$$

In configuration $\mathrm{B} 1$, the principal curvatures at a point of the interface $\Sigma$ when $z=0$ are the constants $\kappa_{1}=2$ and $\kappa_{2}=\frac{1}{2}$. Hence, $H\left(y_{\alpha}\right)=\frac{5}{4}$. We infer

$$
s(\sigma)=\frac{1}{\ln 10}\left(\frac{1}{\ell(\sigma)}-\frac{5}{4}\right) \text {. }
$$

The accuracy of the asymptotic expansion is tested by representing the relative error between numerical and theoretical slopes:

$$
\operatorname{err}(\sigma):=\left|\frac{s(\sigma)-\tilde{s}(\sigma)}{s(\sigma)}\right|
$$

see the table 1 . In order to make clear wether the influence of the curvature is visible in computations, we also display the theoretical curvature ratio

$$
\operatorname{curv\_ ratio}(\sigma):=\frac{\frac{5}{4}}{\frac{1}{\ell(\sigma)}-\frac{5}{4}} .
$$

\begin{tabular}{|l|c|c|c|}
\hline$\sigma$ & 5 & 20 & 80 \\
\hline \hline$\ell(\sigma)$ & 0.103 & 0.0515 & 0.0258 \\
\hline$s(\sigma)$ & 3.67332 & 7.88951 & 16.32188 \\
\hline curv_ratio $(\sigma)$ & 0.148 & 0.069 & 0.033 \\
\hline \hline degree $p$ & 10 & 12 & 16 \\
\hline$n(\sigma)$ & 7 & 6 & 5 \\
\hline$\tilde{s}(\sigma)$ & 3.64686 & 7.87347 & 16.308279 \\
\hline $\operatorname{err}(\sigma)$ & 0.0072 & 0.002 & 0.0008 \\
\hline
\end{tabular}

TABLE 1. Postprocessing in configuration B1 with the mesh $\mathfrak{M}_{3}$

The relative error decreases more than a half when the conductivity is multiplied by 4 . Anyway, this relative error is much smaller than curv_ratio $(\sigma)$, which exhibits numerically the influence of the curvature on the skin depth. We perform similar computations with the mesh $\mathfrak{M}_{6}$ represented in Figure 7, see Table 2, and obtain still better results.

Remark 7.1. A similar postprocessing along the $r$-axis was performed for configuration A, see $[17$, Ch. 8, §8.3.2]. Relative errors $\operatorname{err}(\sigma)$ are still consistent with the expansion (7.1). Non-radial postprocessings was performed along the segment $O L$, when $L$ is the point with a colatitude $\varphi_{0}$ in the interface $\Sigma^{\mathrm{m}}$, see Figure 20: when $\varphi_{0}=\pi / 2-$ $\arctan (1 / 2)$ (i.e. $r_{L}=\sqrt{2}, z_{L}=1 / \sqrt{2}$ ), see [17, Ch. 8, §8.5.1]. Relative errors are again very small. 


\begin{tabular}{|l|c|c|c|}
\hline$\sigma$ & 5 & 20 & 80 \\
\hline \hline degree $p$ & 8 & 12 & 16 \\
\hline$n(\sigma)$ & 13 & 9 & 7 \\
\hline$\tilde{s}(\sigma)$ & 3.64239 & 7.88170 & 16.33051 \\
\hline $\operatorname{err}(\sigma)$ & 0.0084 & 0.001 & 0.0005 \\
\hline
\end{tabular}

TABLE 2. Postprocessing in configuration B1 with the mesh $\mathfrak{M}_{6}$ (see Table 1 for the theoretical values $\ell(\sigma), s(\sigma)$, and curv_ratio $(\sigma)$ )

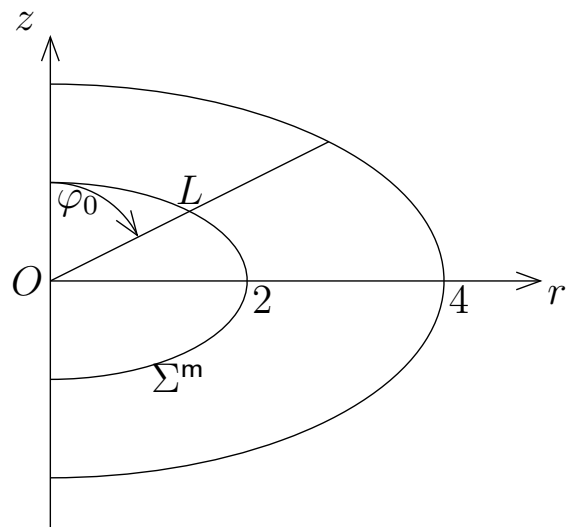

FIGURE 20. In the meridian domain in configuration $\mathrm{B} 1, L$ is the point on $\Sigma^{\mathrm{m}}$ with a colatitude $\varphi_{0}=\pi / 2-\arctan (1 / 2)$

7.2. Configuration A. The mesh of the computational domain is the mesh $\mathfrak{M}_{4}$ represented in Figure 11. We extract values of $\log _{10}\left|\tilde{h}_{\sigma}\right|$ in $\Omega_{-}^{\mathrm{m}}$ along the diagonal axis $r=z$, see Figure 21: here we denote by

$$
\rho:=\sqrt{(1-r)^{2}+(1-z)^{2}},
$$

the distance to the corner point a with coordinates $(r=1, z=1)$, cf. Figure 4 .

When compared with Figure 19, we see that the curves do not exactly behave like lines, which means that the exponential decay is not obvious. In order to measure a possible exponential decay, we define the slopes $\tilde{s}_{i}(\sigma)$ of extracted values $\log _{10}\left|\tilde{\mathrm{h}}_{\sigma}(\rho)\right|$ by:

$$
\tilde{s}_{i}(\sigma):=\frac{\log _{10}\left|\tilde{\mathrm{h}}_{\sigma}\left(r_{i}, z_{i}\right)\right|-\log _{10}\left|\tilde{\mathrm{h}}_{\sigma}\left(z_{i+1}, r_{i+1}\right)\right|}{\rho_{i+1}-\rho_{i}} .
$$

Here, $\rho_{i}$ is the distance to the corner point a defined by $\rho_{i}:=\sqrt{\left(1-r_{i}\right)^{2}+\left(1-z_{i}\right)^{2}}$ with $\left(r_{i}, z_{i}\right)$ the extraction points. We present in the Figure 22 the graph of the slopes $\tilde{s}_{i}(\sigma)$ for each curve on the Figure $21(\sigma=20,80)$. For the sake of comparison, we also represent on the same figure the slopes of Figure 19 corresponding to configuration B1. 

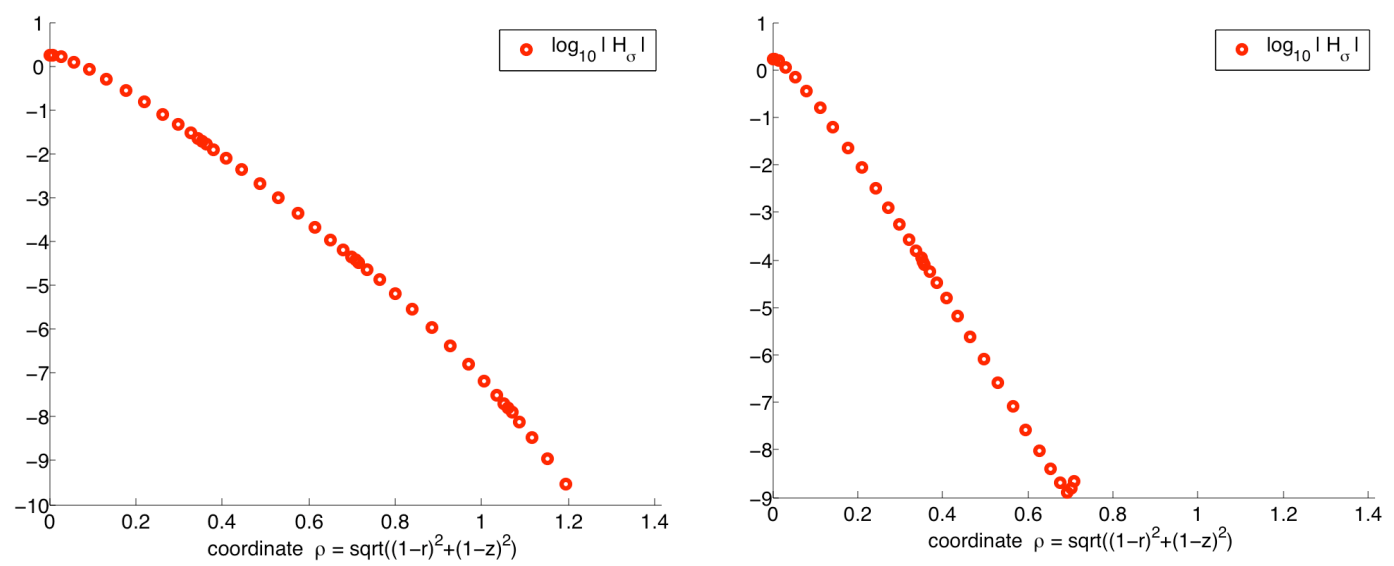

FIGURE 21. In circles, extracted values of $\log _{10}\left|\tilde{\mathrm{h}}_{\sigma}(\rho)\right|, \rho$ is the distance to the corner. On the left $\sigma=20$ (interpolation degree $p=12$ ). On the right, $\sigma=80$ (degree $p=16$ ). Configuration A

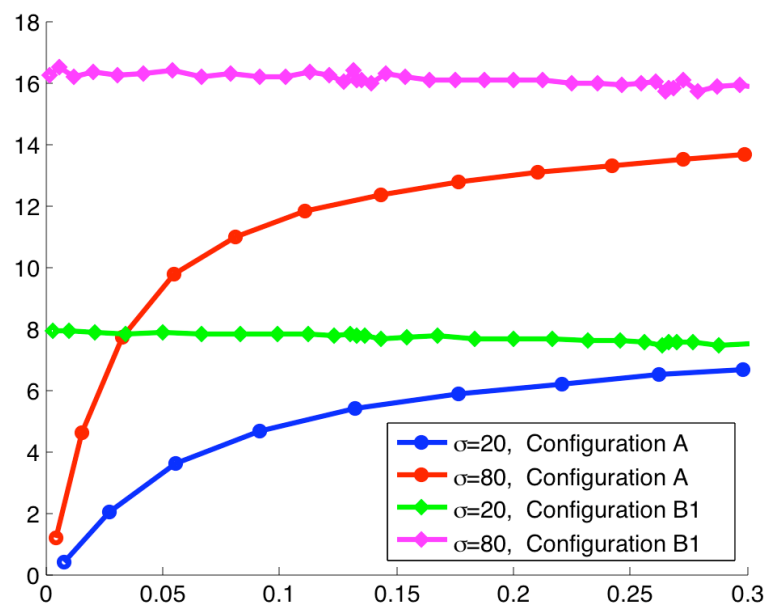

Figure 22. The graphs of the slopes $\tilde{s}_{i}(\sigma)$. Configuration A and B1, and $\sigma=20$ (interpolation degree $p=12$ ), $\sigma=80$ (degree $p=16$ )

Whereas in configuration B1, the slopes clearly converge to a positive limit value as $\rho=y_{3}$ tends to 0 , in configuration A the slopes tend to 0 , which means that, stricto sensu, there is no exponential decay near the corner. Nevertheless we notice that in a region which is further away from the corner, a sort of exponential convergence is restored. This phenomenon is due to the fact that the principal asymptotic contribution inside the conductor is a profile globally defined on an infinite sector $\mathcal{S}$ (of opening $\frac{\pi}{2}$ in the present 
case) solving, instead the 1D problem (4.9) the model Dirichlet problem

$$
\left\{\begin{array}{lll}
\left(\partial_{X}^{2}+\partial_{Y}^{2}\right) \mathfrak{h}_{0}^{\theta}-\lambda^{2} \mathfrak{h}_{0}^{\theta} & =0 & \text { in } \mathcal{S}, \\
\mathfrak{h}_{0}^{\theta} & =\mathrm{h}_{0}^{+}(\mathbf{a}) & \text { on } \partial \mathcal{S} .
\end{array}\right.
$$

\section{CONCLUSION}

Even though addressing axisymmetric configurations for which the Maxwell system can be reduced to one scalar equation, our numerical experiments are in significative accordance with our theoretical results concerning the decay of solutions inside the conductor and their structure in the skin layer. Our asymptotics provide an a priori knowledge on solutions, which can be used for the design of meshes in view of a good quality finite element approximation: The mesh should fit the boundary of the conductor and can be coarse far from its boundary inside the conductor - depending on the skin depth. In this perspective, it is interesting to compare with [16] where an adaptive a posteriori approach based on the $h p$ method has been used for an industrial axisymmetric problem in electromagnetism.

\section{APPENDIX A. ELEMENTS OF PROOF FOR THE MULTISCALE EXPANSION}

Subsequently, we assume Assumption 2.1 on $\omega$ and Assumption 2.3 on the domains. In this framework, Theorem 2.2 gives the existence of $\delta_{0}$ such that for all $\delta \leqslant \delta_{0}$, the problem (2.1)-(2.2) has a unique solution $\left(\mathbf{E}_{(\delta)}, \mathbf{H}_{(\delta)}\right)$ which is denoted by $\left(\mathbf{E}_{(\delta)}^{+}, \mathbf{H}_{(\delta)}^{+}\right)$ in the dielectric part $\Omega_{+}$, and $\left(\mathbf{E}_{(\delta)}^{-}, \mathbf{H}_{(\delta)}^{-}\right)$in the conducting part $\Omega_{-}$. Furthermore, we suppose that the right hand side $\mathbf{j} \in \mathbf{H}_{0}(\operatorname{div}, \Omega)$ is smooth and its support does not meet the conductor domain $\Omega_{-}$.

Recall that $\left(y_{\alpha}, y_{3}\right)$ is a local normal coordinate system to the surface $\Sigma$ in $\mathcal{U}_{-}$, see Figure 2 . The function $\mathbf{y} \mapsto \chi\left(y_{3}\right)$ is a smooth cut-off with support in $\overline{\mathcal{U}}_{-}$and equal to 1 in a smaller tubular neighborhood of $\Sigma$.

Theorem A.1. Under the above assumptions, the solution $\left(\mathbf{E}_{(\delta)}, \mathbf{H}_{(\delta)}\right)$ possesses the asymptotic expansion (see subsection A.7 below for precise estimates):

$$
\mathbf{E}_{(\delta)}^{+}(\mathbf{x}) \approx \sum_{j \geqslant 0} \delta^{j} \mathbf{E}_{j}^{+}(\mathbf{x}) \text { and } \mathbf{H}_{(\delta)}^{+}(\mathbf{x}) \approx \sum_{j \geqslant 0} \delta^{j} \mathbf{H}_{j}^{+}(\mathbf{x}),
$$

$$
\begin{aligned}
\mathbf{E}_{(\delta)}^{-}(\mathbf{x}) & \approx \sum_{j \geqslant 0} \delta^{j} \mathbf{E}_{j}^{-}(\mathbf{x} ; \delta) \quad \text { with } \quad \mathbf{E}_{j}^{-}(\mathbf{x} ; \delta)=\chi\left(y_{3}\right) \underline{\mathfrak{E}}_{j}\left(y_{\beta}, \frac{y_{3}}{\delta}\right), \\
\mathbf{H}_{(\delta)}^{-}(\mathbf{x}) & \approx \sum_{j \geqslant 0} \delta^{j} \mathbf{H}_{j}^{-}(\mathbf{x} ; \delta) \quad \text { with } \quad \mathbf{H}_{j}^{-}(\mathbf{x} ; \delta)=\chi\left(y_{3}\right) \underline{\mathfrak{H}}_{j}\left(y_{\beta}, \frac{y_{3}}{\delta}\right),
\end{aligned}
$$

where $\underline{\mathfrak{E}}_{j}\left(y_{\beta}, \frac{y_{3}}{\delta}\right) \rightarrow 0$ and $\underline{\mathfrak{H}}_{j}\left(y_{\beta}, \frac{y_{3}}{\delta}\right) \rightarrow 0$ when $\frac{y_{3}}{\delta} \rightarrow \infty$. Moreover, for any $j \in \mathbb{N}$, there holds

$$
\mathbf{E}_{j}^{+}, \mathbf{H}_{j}^{+} \in \mathbf{H}\left(\operatorname{curl}, \Omega_{+}\right) \quad \text { and } \quad \underline{\mathfrak{E}}_{j}, \underline{\mathfrak{H}}_{j} \in \mathbf{H}\left(\operatorname{curl}, \Sigma \times \mathbb{R}_{+}\right) .
$$


Hereafter, we present elements of proof of this theorem and details about the terms in asymptotics (A.2)-(A.3). In $§$ A.1, we expand the "magnetic" Maxwell operators in power series of $\delta$ inside the boundary layer $\mathcal{U}_{-}$. We deduce in $\S$ A. 2 the equations satisfied by the magnetic profiles, and derive explicitly the first ones in $\S$ A.3. Then in $\S A .4$ and $\S A .5$, we do the same for the electric profiles. As an alternative, we show how to deduce directly the magnetic profiles from the electrical ones in $\S$ A.6. In $\S$ A.7, we conclude to the validation of the asymptotic expansion with a convergence result.

A.1. Expansion of the operators. Integrating by parts in the magnetic variational formulation (2.7), we find the following Maxwell transmission problem

$$
\begin{cases}\operatorname{curl} \operatorname{curl} \mathbf{H}_{(\delta)}^{+}-\kappa^{2} \mathbf{H}_{(\delta)}^{+}=\operatorname{curl} \mathbf{j} & \text { in } \Omega_{+} \\ \operatorname{curl} \operatorname{curl} \mathbf{H}_{(\delta)}^{-}-\kappa^{2}\left(1+\frac{i}{\delta^{2}}\right) \mathbf{H}_{(\delta)}^{-}=0 & \text { in } \Omega_{-} \\ \operatorname{curl} \mathbf{H}_{(\delta)}^{+} \times \mathbf{n}=\left(1+\frac{i}{\delta^{2}}\right)^{-1} \operatorname{curl} \mathbf{H}_{(\delta)}^{-} \times \mathbf{n} & \text { on } \Sigma \\ \mathbf{H}_{(\delta)}^{+} \times \mathbf{n}=\mathbf{H}_{(\delta)}^{-} \times \mathbf{n} & \text { on } \Sigma \\ \mathbf{H}_{(\delta)}^{+} \times \mathbf{n}=0 & \text { on } \Gamma .\end{cases}
$$

It is important to notice that, since $\kappa \neq 0$, it is a consequence of the above equations that

$$
\operatorname{div} \mathbf{H}_{(\delta)}=0 \text { in } \Omega .
$$

Therefore, we have in particular the extra transmission condition

$$
\mathbf{H}_{(\delta)}^{+} \cdot \mathbf{n}=\mathbf{H}_{(\delta)}^{-} \cdot \mathbf{n} \quad \text { on } \quad \Sigma .
$$

We denote by $\mathbf{L}\left(y_{\alpha}, h ; D_{\alpha}, \partial_{3}^{h}\right)$ the 2 d order Maxwell operator curl curl $-\kappa^{2}\left(1+\frac{i}{\delta^{2}}\right) \mathbb{I}$ set in $\mathcal{U}_{-}$in a normal coordinate system. Here $D_{\alpha}$ is the covariant derivative on the interface $\Sigma$, and $\partial_{3}^{h}$ is the partial derivative with respect to the normal coordinate $y_{3}=h$.

Let $a_{\alpha \beta}(h)$ be the metric tensor of the manifold $\Sigma_{h}$, which is the surface contained in $\Omega_{-}$at a distance $h$ of $\Sigma$, see Figure 2. According to [4, 6], the metric tensor in such a coordinate system writes

$$
a_{\alpha \beta}(h)=a_{\alpha \beta}-2 b_{\alpha \beta} h+b_{\alpha}^{\gamma} b_{\gamma \beta} h^{2},
$$

and its inverse expands in power series of $h$

$$
a^{\alpha \beta}(h)=a^{\alpha \beta}+2 b^{\alpha \beta} h+\mathcal{O}\left(h^{2}\right) .
$$

With this metric, a three-dimensional vector field $\underline{\mathfrak{H}}$ can be split into its normal component $\mathfrak{h}$ and its tangential component that can be alternatively viewed as a vector field $\mathfrak{H}^{\alpha}$ or a one-form field $\mathfrak{H}_{\alpha}$ with the relation

$$
\mathfrak{H}^{\alpha}=a^{\alpha \beta}(h) \mathfrak{H}_{\beta} .
$$

Subsequently, we use a property of the covariant derivative, that it acts on functions like the partial derivative: $D_{\alpha} \mathfrak{h}=\partial_{\alpha} \mathfrak{h}$.

We denote by $\mathbf{T}\left(y_{\alpha}, h ; D_{\alpha}, \partial_{3}^{h}\right)$ the tangent trace operator $\left(1+\frac{i}{\delta^{2}}\right)^{-1} \operatorname{curl} \cdot \times \mathbf{n}$ on $\Sigma$. If $\underline{\mathfrak{H}}=\left(\mathfrak{H}_{\alpha}, \mathfrak{h}\right)$, then

$$
\mathbf{T}\left(y_{\alpha}, h ; D_{\alpha}, \partial_{3}^{h}\right) \underline{\mathfrak{H}}=\left(1+\frac{i}{\delta^{2}}\right)^{-1}\left(\partial_{3}^{h} \mathfrak{H}_{\alpha}-D_{\alpha} \mathfrak{h}\right) d y^{\alpha},
$$


see [17, Ch. 6, eq. (6.87)]. The operators $\mathbf{L}$ and $\mathbf{T}$ expand in power series of $h$ with intrinsic coefficients with respect to $\Sigma$, see [6] for the 3D elasticity operator on a thin shell. We make the scaling $Y_{3}=\delta^{-1} h$ to describe the boundary layer with respect to $\delta$. Then, the three-dimensional harmonic Maxwell operators in $\mathcal{U}_{-}$are written $\mathbf{L}[\delta]$ and $\mathbf{T}[\delta]$. These operators expand in power series of $\delta$ with coefficients intrinsic operators :

$$
\mathbf{L}[\delta]=\delta^{-2} \sum_{n=0}^{\infty} \delta^{n} \mathbf{L}^{n} \quad \text { and } \quad \mathbf{T}[\delta]=\sum_{n=1}^{\infty} \delta^{n} \mathbf{T}^{n} .
$$

We denote by $L_{\alpha}^{n}$ and $T_{\alpha}^{n}$ the surface components of $\mathbf{L}^{n}$ and $\mathbf{T}^{n}$. With the summation convention of repeated two dimensional indices (represented by greek letters), there holds

(A.10) $L_{\alpha}^{0}(\underline{\mathfrak{H}})=-\partial_{3}^{2} \mathfrak{H}_{\alpha}-i \kappa^{2} \mathfrak{H}_{\alpha}$ and $L_{\alpha}^{1}(\underline{\mathfrak{H}})=-2 b_{\alpha}^{\beta} \partial_{3} \mathfrak{H}_{\beta}+\partial_{3} D_{\alpha} \mathfrak{h}+b_{\beta}^{\beta} \partial_{3} \mathfrak{H}_{\alpha}$, and

$$
T_{\alpha}^{n}(\underline{\mathfrak{H}})=\left\{\begin{array}{lll}
(-i)^{p} \partial_{3} \mathfrak{H}_{\alpha} & \text { if } & n=2 p-1 \\
i^{p} \partial_{\alpha} \mathfrak{h} & \text { if } & n=2 p .
\end{array}\right.
$$

Here, $\partial_{3}$ is the partial derivative with respect to $Y_{3}$. We denote by $L_{3}^{n}$ the transverse components of $\mathbf{L}^{n}$. There holds

$$
L_{3}^{0}(\underline{\mathfrak{H}})=-i \kappa^{2} \mathfrak{h} \quad \text { and } \quad L_{3}^{1}(\underline{\mathfrak{H}})=\gamma_{\alpha}^{\alpha}\left(\partial_{3} \underline{\mathfrak{H}}\right)+b_{\beta}^{\beta} \partial_{3} \mathfrak{h},
$$

where $\gamma_{\alpha \beta}(\underline{\mathfrak{H}})=\frac{1}{2}\left(D_{\alpha} \mathfrak{H}_{\beta}+D_{\beta} \mathfrak{H}_{\alpha}\right)-b_{\alpha \beta} \mathfrak{h}$ is the change of metric tensor.

A.2. Equations for the coefficients of the magnetic field. The profiles $\underline{\mathfrak{Y}}_{j}$ and the coefficients $\mathbf{H}_{j}^{+}$of the magnetic field satisfy the following system

$$
\begin{gathered}
\mathbf{L}[\delta] \sum_{j \geqslant 0} \delta^{j} \underline{\mathfrak{H}}_{j}\left(y_{\alpha}, Y_{3}\right)=0 \quad \text { in } \Sigma \times I, \\
\mathbf{T}[\delta] \sum_{j \geqslant 0} \delta^{j} \underline{\mathfrak{Y}}_{j}\left(y_{\alpha}, 0\right)=\sum_{j \geqslant 0} \delta^{j} \operatorname{curl} \mathbf{H}_{j}^{+} \times \mathbf{n} \quad \text { on } \Sigma,
\end{gathered}
$$

where $I=(0,+\infty)$. We perform in (A.13)-(A.14) the identification of terms with the same power in $\delta$. The components of equation (A.13) are the collections of equations

$$
\mathbf{L}^{0}\left(\underline{\mathfrak{H}}_{0}\right)=0, \quad \mathbf{L}^{0}\left(\underline{\mathfrak{H}}_{1}\right)+\mathbf{L}^{1}\left(\underline{\mathfrak{Y}}_{0}\right)=0, \quad \text { and } \sum_{l=0}^{n} \mathbf{L}^{n-l}\left(\underline{\mathfrak{Y}}_{l}\right)=0,
$$

for all $n \geqslant 2$. Similarly, the surface components of the equation (A.14) write

$$
\operatorname{curl} \mathbf{H}_{0}^{+} \times \mathbf{n}=0, \quad \text { and } \quad \sum_{k=1}^{n} \mathbf{T}^{k} \underline{\mathfrak{T}}_{n-k}=\operatorname{curl} \mathbf{H}_{n}^{+} \times \mathbf{n},
$$

for all $n \geqslant 1$. Using the expression of the operator $\mathbf{L}^{0}$, and expanding $\mathbf{H}_{(\delta)}^{+}$in $\Omega_{+}$, we thus see that, according to the system (A.5), the profiles $\underline{\mathfrak{Y}}_{n}=\left(\mathfrak{H}_{n}, \mathfrak{h}_{n}\right)$ and the terms 
$\mathbf{H}_{n}^{+}$have to satisfy, for all $n \geq 0$,

(A.17)

$\left\{\begin{array}{lll}(i) & -\lambda^{2} \mathfrak{h}_{n}=\sum_{j=0}^{n-1} L_{3}^{n-j}\left(\underline{\mathfrak{H}}_{j}\right) & \text { in } \quad \Sigma \times I \\ (i i) & \text { curl curl } \mathbf{H}_{n}^{+}-\kappa^{2} \mathbf{H}_{n}^{+}=\delta_{n}^{0} \operatorname{curl} \mathbf{j} & \text { in } \quad \Omega_{+} \\ (\text {iii }) & \operatorname{curl} \mathbf{H}_{n}^{+} \times \mathbf{n}=\sum_{j=0}^{n-1} \mathbf{T}^{n-j} \underline{\mathfrak{H}}_{j} & \text { on } \quad \Sigma \\ (\text { iv }) & \mathbf{H}_{n}^{+} \times \mathbf{n}=0 \\ (v) & \partial_{3}^{2} \mathfrak{H}_{n, \alpha}-\lambda^{2} \mathfrak{H}_{n, \alpha}=\sum_{j=0}^{n-1} L_{\alpha}^{n-j}\left(\underline{\mathfrak{H}}_{j}\right) & \text { in } \quad \Sigma \times I \\ (v i) & \mathfrak{H}_{n}=\mathbf{n} \times \mathbf{h}_{n} \times \mathbf{n} & \text { on } \Sigma .\end{array}\right.$

where $\lambda=\kappa \mathrm{e}^{-i \pi / 4}$, cf (3.5) (so that $-\lambda^{2}=i \kappa^{2}$ ) and $\mathbf{h}_{n}$ denotes the trace of $\mathbf{H}_{n}^{+}$on $\Sigma$. In (A.17), we use the convention that the sums are 0 when $n=0$. The transmission condition (A.6) implies the extra continuity condition

$$
\mathbf{H}_{n}^{+} \cdot \mathbf{n}=\mathfrak{h}_{n} \quad \text { on } \quad \Sigma .
$$

The set of equations (A.17)-(A.18) allows to determine $\underline{\mathfrak{Y}}_{n}$ and $\mathbf{H}_{n}^{+}$by induction.

A.3. First terms of the magnetic field asymptotics. According to equation $(i)$ in (A.17), the normal component $\mathfrak{h}_{0}$ of the first profile in the conductor vanishes:

$$
\mathfrak{h}_{0}=0,
$$

because $\kappa \neq 0$, thus $\lambda \neq 0$.

Hence, according to (A.17) (ii)-(iv) and (A.18), the first term of the magnetic field in the dielectric region solves Maxwell equations with perfectly conducting conditions on $\Sigma$ :

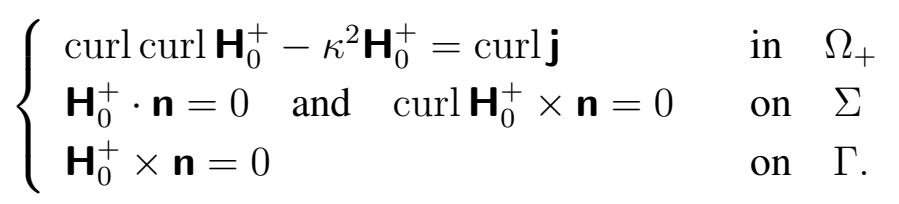

Thus the trace $\mathbf{h}_{0}$ of $\mathbf{H}_{0}^{+}$on the interface $\Sigma$ is tangential.

According to equations $(v)-(v i)$ in (A.17), $\mathfrak{H}_{0}$ satisfies the following ODE

$$
\left\{\begin{array}{lll}
\partial_{3}^{2} \mathfrak{H}_{0}\left(., Y_{3}\right)-\lambda^{2} \mathfrak{H}_{0}\left(., Y_{3}\right) & =0 & \text { for } \quad Y_{3} \in I=(0, \infty) \\
\mathfrak{H}_{0}(., 0) & =\left(\mathbf{n} \times \mathbf{h}_{0}\right) \times \mathbf{n} . &
\end{array}\right.
$$

The unique solution of (A.21) such that $\mathfrak{H}_{0} \rightarrow 0$ when $Y_{3} \rightarrow \infty$, is, with the choice (3.5) for $\lambda$, the tangential field $\mathfrak{H}_{0}\left(y_{\beta}, Y_{3}\right)=\mathbf{h}_{0}\left(y_{\beta}\right) \mathrm{e}^{-\lambda Y_{3}}$. Combining with (A.19), we find that the first profile in the conductor region is exponential with the complex rate $\lambda$ :

$$
\underline{\mathfrak{H}}_{0}\left(y_{\beta}, Y_{3}\right)=\mathbf{h}_{0}\left(y_{\beta}\right) \mathrm{e}^{-\lambda Y_{3}} .
$$


The next term which is determined in the asymptotics is the normal component $\mathfrak{h}_{1}$ of the profile $\underline{\mathfrak{H}}_{1}$ given by equation $(i)$ of (A.17) for $n=1$. We obtain

$$
\mathfrak{h}_{1}\left(y_{\beta}, Y_{3}\right)=\lambda^{-1} D_{\alpha} \mathrm{h}_{0}^{\alpha}\left(y_{\beta}\right) \mathrm{e}^{-\lambda Y_{3}} .
$$

According to (A.17) $(i i)-(i v)$ and (A.18), the next term in the dielectric region solves:

$$
\begin{cases}\text { curl curl } \mathbf{H}_{1}^{+}-\kappa^{2} \mathbf{H}_{1}^{+}=0 & \text { in } \Omega_{+} \\ \mathbf{H}_{1}^{+} \cdot \mathbf{n}=\mathfrak{h}_{1} \quad \text { and } \quad \operatorname{curl} \mathbf{H}_{1}^{+} \times \mathbf{n}=i \lambda \mathbf{h}_{0} & \text { on } \Sigma \\ \mathbf{H}_{1}^{+} \times \mathbf{n}=0 & \text { on } \Gamma .\end{cases}
$$

Recall that $\mathbf{h}_{1}$ is the trace of $\mathbf{H}_{1}^{+}$on the interface $\Sigma$. We denote by $h_{1, \alpha}$ its tangential components. According to equations $(v)-(v i)$ in (A.17) for $n=1, \mathfrak{H}_{1}$ satisfies the following ODE (for $Y_{3} \in I$ )

$$
\left\{\begin{array}{l}
\partial_{3}^{2} \mathfrak{H}_{1, \alpha}\left(., Y_{3}\right)-\lambda^{2} \mathfrak{H}_{1, \alpha}\left(., Y_{3}\right)=-2 b_{\alpha}^{\sigma} \partial_{3} \mathfrak{H}_{0, \sigma}\left(., Y_{3}\right)+b_{\beta}^{\beta} \partial_{3} \mathfrak{H}_{0, \alpha}\left(., Y_{3}\right) \\
\mathfrak{H}_{1, \alpha}(., 0)=\mathrm{h}_{1, \alpha}(., 0)
\end{array}\right.
$$

From (A.22), the unique solution of (A.25) such that $\mathfrak{H}_{1} \rightarrow 0$ when $Y_{3} \rightarrow \infty$ is the profile

$$
\mathfrak{H}_{1, \alpha}\left(y_{\beta}, Y_{3}\right)=\left[\mathrm{h}_{1, \alpha}+Y_{3}\left(H \mathrm{~h}_{0, \alpha}-b_{\alpha}^{\sigma} \mathrm{h}_{0, \sigma}\right)\right]\left(y_{\beta}\right) \mathrm{e}^{-\lambda Y_{3}}, \quad \alpha=1,2 .
$$

Using the relation (A.8) and performing the scaling $h=\delta Y_{3}$ in the previous equation, we obtain for the contravariant components

$$
\mathfrak{H}_{1}^{\alpha}=a^{\alpha \beta} \mathfrak{H}_{1, \beta}+2 Y_{3} b^{\alpha \beta} \mathfrak{H}_{0, \beta} .
$$

From (A.22), and (A.26), the tangential components $\mathfrak{H}_{1}^{\alpha}$ are given by (3.9)

$$
\mathfrak{H}_{1}^{\alpha}\left(y_{\beta}, Y_{3}\right)=\left[\mathrm{h}_{1}^{\alpha}+Y_{3}\left(H \mathrm{~h}_{0}^{\alpha}+b_{\sigma}^{\alpha} \mathrm{h}_{0}^{\sigma}\right)\right]\left(y_{\beta}\right) \mathrm{e}^{-\lambda Y_{3}}, \quad \alpha=1,2 .
$$

Remark A.2. Note that the boundary value problems (A.20) and (A.24) are well-posed. It is a consequence of the spectral Assumption 2.1 on $\omega$.

A.4. Equations for the coefficients of the electric field. The second order Maxwell operator for the electric field writes

$$
\begin{cases}\operatorname{curl} \operatorname{curl} \mathbf{E}_{(\delta)}^{+}-\kappa^{2} \mathbf{E}_{(\delta)}^{+}=i \omega \mu_{0} \mathbf{j} & \text { in } \Omega_{+} \\ \operatorname{curl} \operatorname{curl} \mathbf{E}_{(\delta)}^{-}-\kappa^{2}\left(1+\frac{i}{\delta^{2}}\right) \mathbf{E}_{(\delta)}^{-}=0 & \text { in } \Omega_{-} \\ \operatorname{curl} \mathbf{E}_{(\delta)}^{+} \times \mathbf{n}=\operatorname{curl} \mathbf{E}_{(\delta)}^{-} \times \mathbf{n} & \text { on } \Sigma \\ \mathbf{E}_{(\delta)}^{+} \times \mathbf{n}=\mathbf{E}_{(\delta)}^{-} \times \mathbf{n} & \text { on } \Sigma \\ \mathbf{E}_{(\delta)}^{+} \cdot \mathbf{n}=0 \text { and } \operatorname{curl} \mathbf{E}_{(\delta)}^{+} \times \mathbf{n}=0 & \text { on } \Gamma .\end{cases}
$$

We denote by $\mathbf{B}\left(y_{\alpha}, h ; D_{\alpha}, \partial_{3}^{h}\right)$ the tangent trace operator curl $\cdot \times \mathbf{n}$ on $\Sigma$ in a normal coordinate system. If $\mathfrak{E}=\left(\mathfrak{E}_{\alpha}, \mathfrak{e}\right)$, then

$$
\left(\mathbf{B}\left(y_{\alpha}, h ; D_{\alpha}, \partial_{3}^{h}\right) \mathfrak{E}\right)_{\alpha}=\partial_{3}^{h} \mathfrak{E}{ }_{\alpha}-D_{\alpha} \mathfrak{e}
$$


see $\left[17\right.$, Ch. 3, Prop. 3.36]. We define $\mathbf{B}[\delta]$ the operator obtained from $\mathbf{B}$ in $\mathcal{U}_{-}$after the scaling $Y_{3}=\delta^{-1} h$. This operator expands in power of $\delta$ :

$$
\mathbf{B}[\delta]=\delta^{-1} \mathbf{B}^{0}+\mathbf{B}^{1} .
$$

Recall that $\partial_{3}$ is the partial derivative with respect to $Y_{3}$. Thus, denoting by $B_{\alpha}^{n}$ the surface components of $\mathbf{B}^{n}$, we obtain

$$
B_{\alpha}^{0}(\underline{\mathfrak{E}})=\partial_{3} \mathfrak{E} \alpha \quad \text { and } \quad B_{\alpha}^{1}(\underline{\mathfrak{E}})=-D_{\alpha} \mathfrak{e} .
$$

According to the second and third equations in system (A.27), the profiles $\underline{\mathfrak{E}}_{j}$ and the terms $\mathbf{E}_{j}^{+}$of the electric field satisfy the following system

$$
\begin{gathered}
\mathbf{L}[\delta] \sum_{j \geqslant 0} \delta^{j} \underline{\mathfrak{E}}_{j}\left(y_{\alpha}, Y_{3}\right)=0, \quad \text { in } \quad \Sigma \times I, \\
\mathbf{B}[\delta] \sum_{j \geqslant 0} \delta^{j} \underline{\mathfrak{E}}_{j}\left(y_{\alpha}, 0\right)=\sum_{j \geqslant 0} \delta^{j} \operatorname{curl} \mathbf{E}_{j}^{+} \times \mathbf{n} \text { on } \Sigma .
\end{gathered}
$$

We identify in (A.29)-(A.30) the terms with the same power in $\delta$. The components of the equation (A.29) are collections of equations, similar to the equations (A.15) set for the magnetic field. The surface components of the equation (A.30) write

$$
B_{\alpha}^{0}\left(\underline{\mathfrak{E}}_{0}\right)=0, \quad \text { and } \quad B_{\alpha}^{0}\left(\underline{\mathfrak{E}}_{n+1}\right)+B_{\alpha}^{1}\left(\underline{\mathfrak{E}}_{n}\right)=\left(\operatorname{curl} \mathbf{E}_{n}^{+} \times \mathbf{n}\right)_{\alpha},
$$

for all $n \geqslant 0$.

According to the system (A.27) and (A.28), the profiles $\underline{\mathfrak{E}}_{n}=\left(\mathfrak{E}_{n}, \mathfrak{e}_{n}\right)$ and the terms $\mathbf{E}_{n}^{+}$have to satisfy, for all $n \geqslant 0,\left(\right.$ we recall $\lambda=\kappa \mathrm{e}^{-i \pi / 4}, \operatorname{cf}(3.5)$, so that $\left.-\lambda^{2}=i \kappa^{2}\right)$

$$
\left\{\begin{array}{lll}
(i) & \partial_{3}^{2} \mathfrak{E}_{n, \alpha}-\lambda^{2} \mathfrak{E}_{n, \alpha}=\sum_{j=0}^{n-1} L_{\alpha}^{n-j}\left(\mathfrak{E}_{j}\right) & \text { in } \Sigma \times I \\
(i i) & \partial_{3} \mathfrak{E}_{n, \alpha}=D_{\alpha} \mathfrak{e}_{n-1}+\left(\operatorname{curl} \mathbf{E}_{n-1}^{+} \times \mathbf{n}\right)_{\alpha} & \text { on } \Sigma \\
(i i i) & -\lambda^{2} \mathfrak{e}_{n}=\sum_{j=0}^{n-1} L_{3}^{n-j}\left(\mathfrak{E}_{j}\right) & \text { in } \Sigma \times I \\
(i v) & \operatorname{curlcurl} \mathbf{E}_{n}^{+}-\kappa^{2} \mathbf{E}_{n}^{+}=\delta_{n}^{0} i \omega \mu_{0} \mathbf{j} & \text { in } \Omega_{+} \\
(v) & \mathbf{E}_{n}^{+} \times \mathbf{n}=\mathfrak{E}_{n} \times \mathbf{n} & \text { on } \Sigma \\
(v i) & \mathbf{E}_{n}^{+} \cdot \mathbf{n}=0 \text { and } \operatorname{curl} \mathbf{E}_{n}^{+} \times \mathbf{n}=0 & \text { on } \Gamma .
\end{array}\right.
$$

Hereafter, we determine the terms $\underline{\mathfrak{E}}_{n}$ and $\mathbf{E}_{n}^{+}$by induction.

A.5. First terms of the asymptotics for the electric field. According to equations $(i)$ (ii) in system (A.32) for $n=0, \mathfrak{E}_{0}$ satisfies the following ODE

$$
\begin{cases}\partial_{3}^{2} \mathfrak{E}_{0}\left(., Y_{3}\right)-\lambda^{2} \mathfrak{E}_{0}\left(., Y_{3}\right) & =0 \quad \text { for } \quad Y_{3} \in I \\ \partial_{3} \mathfrak{E}_{0}(., 0) & =0\end{cases}
$$

The unique solution of (A.33) such that $\mathfrak{E}_{0} \rightarrow 0$ when $Y_{3} \rightarrow \infty$, is $\mathfrak{E}_{0}=0$. From equation $($ iii $)$ in system (A.32) for $n=0$, there holds $\mathfrak{e}_{0}=0$. We infer

$$
\underline{\mathfrak{E}}_{0}\left(y_{\beta}, Y_{3}\right)=0 .
$$


From equations $(i v)-(v i)$ in (A.32) for $n=0$, and from (A.34), the first asymptotic of the electric field in the dielectric part solves the following problem

$$
\begin{cases}\text { curlcurl } \mathbf{E}_{0}^{+}-\kappa^{2} \mathbf{E}_{0}^{+}=i \omega \mu_{0} \mathbf{j} & \text { in } \Omega_{+} \\ \mathbf{E}_{0}^{+} \times \mathbf{n}=0 & \text { on } \Sigma \\ \mathbf{E}_{0}^{+} \cdot \mathbf{n}=0 \text { and } \quad \operatorname{curl} \mathbf{E}_{0}^{+} \times \mathbf{n}=0 & \text { on } \Gamma .\end{cases}
$$

According to the spectral Assumption 2.1, this boundary value problem is well-posed.

The next term determined in the asymptotic expansion is $\mathfrak{E}_{1}$. From equations $(i)-(i i)$ in (A.32) for $n=1, \mathfrak{E}_{1}$ satisfies for $Y_{3} \in I$

$$
\begin{cases}\partial_{3}^{2} \mathfrak{E}_{1}\left(., Y_{3}\right)-\lambda^{2} \mathfrak{E}_{1}\left(., Y_{3}\right) & =0 \\ \partial_{3} \mathfrak{E}_{1}(., 0) & =\left(\operatorname{curl} \mathbf{E}_{0}^{+} \times \mathbf{n}\right)(., 0) .\end{cases}
$$

We denote by $\mathbf{j}_{k}\left(y_{\beta}\right)=\lambda^{-1}\left(\operatorname{curl} \mathbf{E}_{k}^{+} \times \mathbf{n}\right)\left(y_{\beta}, 0\right)$ for $k=0,1$. Hence,

$$
\mathfrak{E}_{1}\left(y_{\beta}, Y_{3}\right)=-\mathbf{j}_{0}\left(y_{\beta}\right) \mathrm{e}^{-\lambda Y_{3}} \text {. }
$$

From equation ( iii $)$ in (A.32) for $n=1$, and from (A.34), we obtain $\mathfrak{e}_{1}=0$. From equations $(i v)-(v i)$ in (A.32) for $n=1$, and from (A.35), the asymptotic of order 1 for the electric field in the dielectric part solves :

$$
\begin{cases}\text { curlcurl } \mathbf{E}_{1}^{+}-\kappa^{2} \mathbf{E}_{1}^{+}=0 & \text { in } \Omega_{+} \\ \mathbf{E}_{1}^{+} \times \mathbf{n}=-\mathbf{j}_{0} \times \mathbf{n} & \text { on } \Sigma \\ \mathbf{E}_{1}^{+} \cdot \mathbf{n}=0 \text { and } \operatorname{curl} \mathbf{E}_{1}^{+} \times \mathbf{n}=0 & \text { on } \Gamma .\end{cases}
$$

Then, from equations $(i)$ - $(i i)$ in (A.32) for $n=2, \mathfrak{E}_{2, \alpha}$ solves the ODE for $Y_{3} \in I$ :

$$
\begin{cases}\partial_{3}^{2} \mathfrak{E}_{2, \alpha}\left(., Y_{3}\right)-\lambda^{2} \mathfrak{E}_{2, \alpha}\left(., Y_{3}\right) & =-2 b_{\alpha}^{\sigma} \partial_{3} \mathfrak{E}_{1, \sigma}\left(., Y_{3}\right)+b_{\beta}^{\beta} \partial_{3} \mathfrak{E}_{1, \alpha}\left(., Y_{3}\right) \\ \partial_{3} \mathfrak{E}_{2, \alpha}(., 0) & =\left(\operatorname{curl} \mathbf{E}_{1}^{+} \times \mathbf{n}\right)_{\alpha}(., 0) .\end{cases}
$$

We denote by $\mathbf{j}_{k, \alpha}$ the surface components of $\mathbf{j}_{k}$, for $k=0,1$. We obtain

$$
\mathfrak{E}_{2, \alpha}\left(y_{\beta}, Y_{3}\right)=\left[-\mathrm{j}_{1, \alpha}+\left(\lambda^{-1}+Y_{3}\right)\left(b_{\alpha}^{\sigma} \mathrm{j}_{0, \sigma}-H \mathrm{j}_{0, \alpha}\right)\right]\left(y_{\beta}\right) \mathrm{e}^{-\lambda Y_{3}} .
$$

From equation $($ iii) in (A.32) for $n=2$, we infer

$$
\mathfrak{e}_{2}\left(y_{\beta}, Y_{3}\right)=-\lambda^{-1} D_{\alpha} \mathrm{j}_{0}^{\alpha}\left(y_{\beta}\right) \mathrm{e}^{-\lambda Y_{3}} .
$$

A.6. From $\mathbf{E}$ to $\mathbf{H}$ in the conducting part. An alternative way of calculating the magnetic profiles is to deduce them from the electric ones by means of a normal parameterization of the intrinsic curl operator, see [17, Ch. 3]. Let $\mathfrak{E}=\left(\mathfrak{E}_{\alpha}, \mathfrak{e}\right)$ be a 1-form fields in $\mathcal{U}_{-}$. There holds:

$$
\text { (A.37) }(\nabla \times \underline{\mathfrak{E}})^{\alpha}=\epsilon^{3 \beta \alpha}\left(\partial_{3}^{h} \mathfrak{E}_{\beta}-\partial_{\beta} \mathfrak{e}\right) \quad \text { and } \quad(\nabla \times \underline{\mathfrak{E}})^{3}=\epsilon^{3 \alpha \beta} D_{\alpha}^{h} \mathfrak{E}_{\beta} \quad \text { on } \quad \Sigma_{h} .
$$

Here, $D_{\alpha}^{h}$ is the covariant derivative on $\Sigma_{h}$, and $\epsilon$ is the Levi-Civita tensor, see [10, 7]. The contravariant components $\epsilon^{i j k}$ of $\epsilon$ depend on the normal coordinate $h$, and write in a normal coordinate system

$$
\epsilon^{i j k}=\left(\operatorname{det} a_{\alpha \beta}(h)\right)^{-1 / 2} \epsilon_{0}(i, j, k) .
$$


Here, $a_{\alpha \beta}(h)$ is the metric tensor of the manifold $\Sigma_{h}$, see (A.7). The indices $i, j, k \in$ $\{1,2,3\}$, and $\epsilon_{0}(i, j, k)$ equals 1 when $(i, j, k)$ is an even circular permutation, and equals -1 when $(i, j, k)$ is an odd circular permutation, and $\epsilon_{0}(i, j, k)=0$ otherwise.

Remark A.3. Let $a=\operatorname{det}\left(a_{\alpha \beta}\right)$, and recall that $b_{\nu}^{\nu}=2 H$. Then using (A.7) we obtain

$$
\epsilon^{i j k}=a^{-1 / 2}\left(1+2 H h+O\left(h^{2}\right)\right) \epsilon_{0}(i, j, k) .
$$

We make the scaling $h=\delta Y_{3}$ and expand equations (A.37) in power series of $\delta$ :

$$
\begin{aligned}
& (\nabla \times \underline{\mathfrak{E}})^{\alpha}=\delta^{-1} j^{\beta \alpha} \partial_{3} \mathfrak{E}_{\beta}+j^{\beta \alpha}\left(2 H Y_{3} \partial_{3} \mathfrak{E}_{\beta}-\partial_{\beta} \mathfrak{e}\right)+\mathcal{O}(\delta), \\
& (\nabla \times \underline{\mathfrak{E}})^{3}=j^{\alpha \beta} D_{\alpha} \mathfrak{E}_{\beta}+\delta Y_{3} j^{\alpha \beta}\left(2 H D_{\alpha} \mathfrak{E}_{\beta}+\mathfrak{E}_{\nu} D_{\alpha} b_{\beta}^{\nu}\right)+\mathcal{O}\left(\delta^{2}\right) .
\end{aligned}
$$

Here,

$$
j^{\alpha \beta}=a^{-1 / 2} \epsilon_{0}(\alpha, \beta, 3) .
$$

From the expansions (A.2) and (A.1) combined with Faraday's law (2.1) written in normal coordinates, we obtain the profiles $\mathfrak{\mathfrak { Y }}_{j}$ in the expansion (A.3) of the magnetic field from the profiles $\mathfrak{E}_{j}$ of the electrical field. In particular, from (A.34), (A.35), and (A.36), we obtain explicitly the first terms $\underline{\mathfrak{H}}_{0}$ and $\underline{\mathfrak{H}}_{1}$, see (A.22), (3.9), and (A.23).

A.7. Convergence result. The validation of the asymptotic expansion (A.1), (A.2),(A.3), and (A.4), consists in proving estimates for remainders defined as

$$
\mathbf{R}_{m ; \delta}^{\mathbf{E}}=\mathbf{E}_{(\delta)}-\sum_{j=0}^{m} \delta^{j} \mathbf{E}_{j} \quad \text { and } \quad \mathbf{R}_{m ; \delta}^{\mathbf{H}}=\mathbf{H}_{(\delta)}-\sum_{j=0}^{m} \delta^{j} \mathbf{H}_{j} \quad \text { in } \quad \Omega .
$$

By construction of the terms $\mathbf{E}_{j}=\left(\mathbf{E}_{j}^{+}, \mathbf{E}_{j}^{-}\right)$in the dielectric and conductor parts, the remainders $\mathbf{R}_{m ; \delta}^{\mathrm{E}}$ satisfy the assumption of [3, Th. 5.1], which is an estimate on the right hand side when the Maxwell operator is applied to $\mathbf{R}_{m ; \delta}^{\mathbf{E}}$.

Thus [3, Th. 5.1] yields that for all $m \in \mathbb{N}$, and $\delta \in\left(0, \delta_{0}\right)$, there holds the optimal estimate

$$
\begin{aligned}
\left\|\mathbf{R}_{m ; \delta}^{\mathbf{E},+}\right\|_{0, \Omega_{+}}+ & \left\|\operatorname{curl} \mathbf{R}_{m ; \delta}^{\mathbf{E},+}\right\|_{0, \Omega_{+}} \\
& +\delta^{-\frac{1}{2}}\left\|\mathbf{R}_{m ; \delta}^{\mathbf{E},-}\right\|_{0, \Omega_{-}}+\delta^{\frac{1}{2}}\left\|\operatorname{curl} \mathbf{R}_{m ; \delta}^{\mathbf{E},-}\right\|_{0, \Omega_{-}} \leqslant C_{m} \delta^{m+1} .
\end{aligned}
$$

Using Maxwell equations (2.1), we can deduce a similar estimate for $\mathbf{R}_{m ; \delta}^{\mathbf{H}}$ : We have

$$
\mathbf{R}_{m ; \delta}^{\mathbf{H},+}=\left(i \omega \mu_{0}\right)^{-1} \operatorname{curl} \mathbf{R}_{m ; \delta}^{\mathbf{E},+} \quad \text { and } \quad \operatorname{curl} \mathbf{R}_{m ; \delta}^{\mathbf{H},+}=\left(i \omega \mu_{0}\right)^{-1} \kappa^{2} \mathbf{R}_{m ; \delta}^{\mathbf{E},+} .
$$

Hence, according to (A.41), we infer

$$
\left\|\mathbf{R}_{m ; \delta}^{\mathbf{H},+}\right\|_{0, \Omega_{+}}+\left\|\operatorname{curl} \mathbf{R}_{m ; \delta}^{\mathbf{H},+}\right\|_{0, \Omega_{+}} \leqslant c_{m} \delta^{m+1} .
$$

In $\Omega_{-}$, the presence of profiles in the expansion prevents to link $\mathbf{R}_{m ; \delta}^{\mathbf{H},-}$ and $\mathbf{R}_{m ; \delta}^{\mathbf{E},-}$ via the Maxwell equations in a similar way as (A.42). Nevertheless, there holds also uniform estimates for $\mathbf{R}_{m ; \delta}^{\mathbf{H},-}$ :

$$
\delta^{-\frac{1}{2}}\left\|\mathbf{R}_{m ; \delta}^{\mathbf{H},-}\right\|_{0, \Omega_{-}}+\delta^{\frac{1}{2}}\left\|\operatorname{curl} \mathbf{R}_{m ; \delta}^{\mathbf{H},-}\right\|_{0, \Omega_{-}} \leqslant C_{m}^{\prime} \delta^{m+1} .
$$




\section{APPENDIX B. THE MULTISCALE EXPANSION OF THE ORTHORADIAL COMPONENT}

We denote by $\left(\overrightarrow{e_{r}}, \overrightarrow{e_{\theta}}, \overrightarrow{e_{z}}\right)$ the basis associated with the cylindric coordinates $(r, \theta, z)$.

B.1. Expansion of the operators. In the basis $\left(\overrightarrow{e_{r}}, \overrightarrow{e_{z}}\right)$, recall that $(r(\xi), z(\xi))=\boldsymbol{\tau}(\xi)$, $\xi \in(0, L)$ is an arc-length coordinate on the interface $\Sigma^{\mathrm{m}}$, and $\left(\xi, h=y_{3}\right)$ is the associate normal coordinate system, see $\S 4$.2.2. The normal vector $\mathbf{n}(\xi)$ at the point $\boldsymbol{\tau}(\xi)$ writes

$$
\mathbf{n}(\xi)=\left(-z^{\prime}(\xi), r^{\prime}(\xi)\right) .
$$

Hence, the tubular neighborhood $\mathcal{U}_{-}^{\mathrm{m}}$ of $\Sigma^{\mathrm{m}}$ inside $\Omega_{-}^{\mathrm{m}}$ is represented thanks to the parameterization

$$
\Psi:(\xi, h) \longmapsto(r, z),
$$

$$
\text { where } r=r(\xi)-h z^{\prime}(\xi), \quad \text { and } \quad z=z(\xi)+h r^{\prime}(\xi) \text {. }
$$

Remark B.1. The curvature $k(\xi)$ at the point $\boldsymbol{\tau}(\xi)$ is defined by

$$
k(\xi)=\left(r^{\prime} z^{\prime \prime}-z^{\prime} r^{\prime \prime}\right)(\xi) .
$$

For $h_{0}<1 /\|k\|_{\infty}$ the change of coordinates $\Psi$ is a $\mathcal{C}^{\infty}$-diffeomorphism from the cylinder $\mathbb{T}_{L} \times\left[0, h_{0}\right)$ into $\mathcal{U}_{-}^{\mathrm{m}}$ :

$$
\mathcal{U}_{-}^{\mathrm{m}}=\Psi\left(\mathbb{T}_{L} \times\left[0, h_{0}\right)\right) .
$$

Thanks to (B.1), we obtain

$$
\left(\begin{array}{c}
\partial_{r} \\
\partial_{z}
\end{array}\right)=(1-h k(\xi))^{-1}\left(\begin{array}{cc}
r^{\prime} & -\left(z^{\prime}+h r^{\prime \prime}\right) \\
z^{\prime} & \left(r^{\prime}-h z^{\prime \prime}\right)
\end{array}\right)\left(\begin{array}{c}
\partial_{\xi} \\
\partial_{h}
\end{array}\right) .
$$

To perform the formal expansion of $h_{\delta}^{-}$solution of (4.5) in $\Omega_{-}^{\mathrm{m}}$, we first use the change of variables $\Psi$ in order to write the equations in the cylinder $\mathbb{T}_{L} \times\left[0, h_{0}\right)$. We then perform the rescaling

$$
Y=\delta^{-1} h
$$

in the equations set in $\Omega_{-}^{\mathrm{m}}$ and $\Sigma^{\mathrm{m}}$ in order to make appear the small parameter $\delta$ in the equations. Actually $\delta$ appears in the equations set in $\Omega_{-}$through the expression of the operator $\left(1+\frac{i}{\delta^{2}}\right)^{-1} \mathrm{D}$, where $\mathrm{D}$ is defined by (4.7). We obtain the formal expansion:

$$
\mathrm{D}=\delta^{-2}\left[\partial_{Y}^{2}+\delta \mathrm{D}_{1}+\delta^{2} \mathrm{R}_{\delta}\right]
$$

where $\mathrm{D}_{1}\left(\xi, Y ; \partial_{\xi}, \partial_{Y}\right)=-\left(k(\xi)+\frac{z^{\prime}}{r}(\xi)\right) \partial_{Y}$, and $\mathrm{R}_{\delta}$ is an operator, which has smooth coefficients in $\xi$ and $Y$, bounded in $\delta$. Hence, we obtain

$$
i\left(1+\frac{i}{\delta^{2}}\right)^{-1} \mathrm{D}+\kappa^{2} \mathbb{I}=\mathrm{A}_{0}+\delta \mathrm{A}_{1}+\delta^{2} \mathrm{Q}_{\delta},
$$

with $\mathrm{A}_{0}=\partial_{Y}^{2}-\lambda^{2} \mathbb{I}$ and $\mathrm{A}_{1}=-\left(k+\frac{z^{\prime}}{r}\right)(\xi) \partial_{Y}$. Similarly, there holds

$$
\mathrm{B}=\delta^{-1} \partial_{Y}-\frac{z^{\prime}}{r}(\xi)
$$


on the interface $\Sigma^{m}$, and

$$
\left(1+\frac{i}{\delta^{2}}\right)^{-1} \mathrm{~B}=-i \delta \partial_{Y}+\delta^{2} \mathrm{P}_{\delta}
$$

Note that these expansions correspond to the expansions (A.9) in orthoradial symmetry.

B.2. From the 3D asymptotic expansion to the 1D expansion. In this subsection, we show that we can obtain the profiles $\mathfrak{h}_{0}^{\theta}$, and $\mathfrak{h}_{1}^{\theta}$ defined by (4.10) and (4.13) from the general profiles $\mathfrak{H}_{0}^{\alpha}$ and $\mathfrak{H}_{1}^{\alpha}$ defined above.

We set $\left(y_{\alpha}\right):=(\xi, \theta)$ a coordinate system on $\Sigma$. Thus, the normal coordinate system $\left(y_{\alpha}, h\right)$ on $\mathcal{U}_{-}$is induced by the normal coordinate system $(\xi, h)$ on $\mathcal{U}_{-}^{\mathrm{m}}$. The tubular neighborhood $\mathcal{U}_{-}$is parameterized by

$$
\Phi:\left(y_{\alpha}, h\right) \longmapsto(r \cos \theta, r \sin \theta, z),
$$

where $r$ and $z$ are defined by (B.1). The associated tangent coordinate vector fields are

$$
\begin{gathered}
\mathbf{x}_{1}(h)=\left(r^{\prime}(\xi)-h z^{\prime \prime}(\xi)\right) \overrightarrow{e_{r}}+\left(z^{\prime}(\xi)+h r^{\prime \prime}(\xi)\right) \overrightarrow{e_{z}}, \\
\mathbf{x}_{2}(h)=\left(r(\xi)-h z^{\prime}(\xi)\right) \overrightarrow{e_{\theta}} .
\end{gathered}
$$

The normal coordinate vector field is $\mathbf{x}_{3}(h)=\mathbf{n}(\xi)=-z^{\prime}(\xi) \overrightarrow{e_{r}}+r^{\prime}(\xi) \overrightarrow{e_{z}}$.

A vector field $\underline{\mathfrak{H}}:\left(y_{\alpha}, h\right) \mapsto \underline{\mathfrak{H}}\left(y_{\alpha}, h\right)$ in $\mathcal{U}_{-}$writes (here $\left.\alpha=1,2\right)$

$$
\underline{\mathfrak{Y}}=\mathfrak{H}^{\alpha} \mathbf{x}_{\alpha}(h)+\mathfrak{h}^{\theta} \mathbf{n},
$$

and corresponds to the magnetic field in cylindric components

$$
\breve{\mathbf{H}}(r, \theta, z)=H_{r}(r, \theta, z) \overrightarrow{e_{r}}+H_{\theta}(r, \theta, z) \overrightarrow{e_{\theta}}+H_{z}(r, \theta, z) \overrightarrow{e_{z}} .
$$

Using (B.5) we easily obtain

$$
\left(r(\xi)-h z^{\prime}(\xi)\right) \mathfrak{H}^{2}=H_{\theta}
$$

and introducing the stretched variable $Y=\delta^{-1} h$, it follows

$$
\left(r(\xi)-\delta Y z^{\prime}(\xi)\right) \mathfrak{H}^{2}=H_{\theta}
$$

We insert the following expansions

$$
\begin{gathered}
\mathfrak{H}^{2}=\mathfrak{H}_{0}^{2}\left(y_{\alpha}, Y\right)+\delta \mathfrak{H}_{1}^{2}\left(y_{\alpha}, Y\right)+\mathcal{O}\left(\delta^{2}\right), \\
H_{\theta}=\mathfrak{h}_{0}^{\theta}(\xi, Y)+\delta \mathfrak{h}_{1}^{\theta}(\xi, Y)+\mathcal{O}\left(\delta^{2}\right)
\end{gathered}
$$

in equation (B.6). Then we perform the identification of terms with the same power in $\delta$. We obtain the equations

$$
\begin{gathered}
r(\xi) \mathfrak{H}_{0}^{2}=\mathfrak{h}_{0}^{\theta}, \\
r(\xi) \mathfrak{H}_{1}^{2}-Y z^{\prime}(\xi) \mathfrak{H}_{0}^{2}=\mathfrak{h}_{1}^{\theta} .
\end{gathered}
$$

According to (A.22), and (3.9) for $\alpha=2$, there holds

$$
r(\xi) \mathfrak{H}_{1}^{2}-Y z^{\prime}(\xi) \mathfrak{H}_{0}^{2}=r(\xi)\left[\mathrm{h}_{1}^{2}+Y\left(H \mathrm{~h}_{0}^{2}+b_{\sigma}^{2} \mathrm{~h}_{0}^{\sigma}-\frac{z^{\prime}}{r} \mathrm{~h}_{0}^{2}\right)\right] \mathrm{e}^{-\lambda Y} .
$$


Lemma B.2. The main curvatures $\kappa_{1}, \kappa_{2}$ of the interface $\Sigma$, and its mean curvature $H=\frac{1}{2}\left(\kappa_{1}+\kappa_{2}\right)$ are

$$
\kappa_{1}=k, \quad \kappa_{2}=\frac{z^{\prime}}{r}, \quad \text { and } \quad H=\frac{1}{2}\left(k+\frac{z^{\prime}}{r}\right) .
$$

This result together with (B.9) yields

$$
r(\xi) \mathfrak{H}_{1}^{2}-Y z^{\prime}(\xi) \mathfrak{H}_{0}^{2}=r(\xi)\left[\mathrm{h}_{1}^{2}+\frac{Y}{2}\left(k+\frac{z^{\prime}}{r}\right)(\xi) \mathrm{h}_{0}^{2}\right] \mathrm{e}^{-\lambda Y} .
$$

We set $r(\xi) \mathbf{h}_{j}^{2}\left(y_{\beta}\right)=\mathbf{h}_{j}^{+}(\boldsymbol{\tau}(\xi))$, for $j=0,1$. Then, we infer (4.10)-(4.13) from the equations (A.22)-(3.9).

B.3. Proof of the lemma B.2. The main curvatures are defined by $\kappa_{1}=b_{1}^{1}, \kappa_{2}=b_{2}^{2}$, where $b_{\alpha \beta}$ is the curvature tensor. In this coordinate system on $\mathcal{U}_{-}$, the metric is defined by

$$
g_{i j}(h)=\left\langle\mathbf{x}_{i}(h), \mathbf{x}_{j}(h)\right\rangle_{\mathbb{R}^{3}} .
$$

From (B.4)-(B.5), we obtain

$$
g_{i j}(h)=\left(\begin{array}{ccc}
\left(r^{\prime}(\xi)-h z^{\prime \prime}(\xi)\right)^{2}+\left(z^{\prime}(\xi)+h r^{\prime \prime}(\xi)\right)^{2} & 0 & 0 \\
0 & \left(r(\xi)-h z^{\prime}(\xi)\right)^{2} & 0 \\
0 & 0 & 1
\end{array}\right)
$$

Recall that

$$
g_{\alpha \beta}(h)=a_{\alpha \beta}-2 b_{\alpha \beta} h+b_{\alpha}^{\gamma} b_{\gamma \beta} h^{2} .
$$

Hence, the curvature tensor on the interface $\Sigma$ is diagonal, and its diagonal components write

$$
b_{11}=k \quad \text { and } \quad b_{22}=r z^{\prime},
$$

where $k=k(\xi)$ is defined by (B.2). The inverse of the metric tensor $a_{\alpha \beta}$ in $\Sigma$ is diagonal and there holds

$$
a^{11}=1 \text { and } a^{22}=r^{-2} .
$$

From (B.10), we deduce the lemma. 


\section{REFERENCES}

[1] C. Bernardi, M. Dauge, Y. Maday. Spectral methods for axisymmetric domains, volume 3 of Series in Applied Mathematics (Paris). Gauthier-Villars, Éditions Scientifiques et Médicales Elsevier, Paris 1999. Numerical algorithms and tests due to Mejdi Azaïez.

[2] P. Boissoles. Problèmes mathématiques et numériques issus de l'imagerie par résonance magnétique nucléaire. $\mathrm{PhD}$ thesis, Université Rennes 12005.

[3] G. Caloz, M. Dauge, V. PÉRON. Uniform estimates for transmission problems with high contrast in heat conduction and electromagnetism. Journal of Mathematical Analysis and Applications 370(2) (2010) 555-572.

[4] M. DAuge, E. FAOU, V. PÉRON. Comportement asymptotique à haute conductivité de l'épaisseur de peau en électromagnétisme. C. R. Acad. Sci. Paris Sér. I Math. 348(7-8) (2010) 385-390.

[5] M. Dauge, V. PÉron, C. Poignard. Asymptotic expansion for the solution of a stiff transmission problem in electromagnetism with a singular interface. (In preparation), 2010.

[6] E. FAOU. Elasticity on a thin shell: formal series solution. Asymptot. Anal. 31(3-4) (2002) 317-361.

[7] H. Goldstein. Classical mechanics. Addison-Wesley Publishing Co., Reading, Mass., second edition 1980. Addison-Wesley Series in Physics.

[8] H. Haddar, P. Joly, H.-M. NGUYEN. Generalized impedance boundary conditions for scattering problems from strongly absorbing obstacles: the case of Maxwell's equations. Math. Models Methods Appl. Sci. 18(10) (2008) 1787-1827.

[9] R. HiptmaiR, P. D. Ledger. Computation of resonant modes for axisymmetric Maxwell cavities using $h p$-version edge finite elements. Internat. J. Numer. Methods Engrg. 62(12) (2005) 1652-1676.

[10] T. Levi-Civita. The absolute differential calculus. Dover Phoenix Editions. Dover Publications Inc., Mineola, NY 2005. Calculus of tensors, Translated from the Italian by Marjorie Long, Edited by Enrico Persico, Reprint of the 1926 translation.

[11] R. C. MACCAmy, E. STEPHAN. Solution procedures for three-dimensional eddy current problems. J. Math. Anal. Appl. 101(2) (1984) 348-379.

[12] R. C. MacCamy, E. Stephan. A skin effect approximation for eddy current problems. Arch. Rational Mech. Anal. 90(1) (1985) 87-98.

[13] D. Martin. Mélina, bibliothèque de calculs éléments finis. Source code. http://anum-maths.univrennes 1.fr/melina, 1990-2010.

[14] P. NAGHDi. Foundations of elastic shell theory. In Progress in Solid Mechanics, Vol. IV, pages 1-90. North-Holland, Amsterdam 1963.

[15] B. NKEMZI. On the solution of Maxwell's equations in axisymmetric domains with edges. ZAMM Z. Angew. Math. Mech. 85(8) (2005) 571-592.

[16] D. Pardo, L. Demkowicz, C. Torres-Verdín, M. Paszynski. Two-dimensional highaccuracy simulation of resistivity logging-while-drilling (LWD) measurements using a self-adaptive goal-oriented $h p$ finite element method. SIAM J. Appl. Math. 66(6) (2006) 2085-2106.

[17] V. PÉRON. Modélisation mathématique de phénomènes électromagnétiques dans des matériaux à fort contraste. $\mathrm{PhD}$ thesis, Université Rennes 1 2009. http://tel.archives-ouvertes.fr/tel-00421736/fr/.

[18] S. M. Rytov. Calcul du skin effect par la méthode des perturbations. Journal of Physics 11(3) (1940) 233-242.

[19] C. Schwab, M. SuRI. The $p$ and $h p$ versions of the finite element method for problems with boundary layers. Math. Comp. 65(216) (1996) 1403-1429.

[20] E. STEPHAN. Solution procedures for interface problems in acoustics and electromagnetics. In Theoretical acoustics and numerical techniques, volume 277 of CISM Courses and Lectures, pages 291-348. Springer, Vienna 1983. 